\title{
The Methods of Numerical Time Integration of One-dimensional Linear Equations and Their Inherited Errors
}

\author{
By K. Miyakoda \\ Geophysical Institute, Tokyo University \\ (Manuscript received 29 July 1960)
}

\begin{abstract}
For solving one-dimensional linear partial differential equations numerically, several methods are studied on the accuracy, the stability and the inherited errors of solutions. First, the time extrapolation scheme is discussed, and the 3 time-level method (the usual centered difference) and one of the 4-level methods are concluded to give satisfactory results. In particular, the latter is numerically stable even for the equation of frictional type. Second, concerning the formulas of approximating the space derivative by finite difference, the 3 gridpoint and the 5 gridpoint methods are examined. The differential equations treated involves the advection term, where the advecting flow is spatially variable. Namely, the term is consisted of the product of two space-dependent quantities, which produces new waves by their interaction. It is shown that the usual computing method, i.e., the "3-3 pt" method (3 levels for time and 3 points for space difference) gives arise the small scale errors, which turn out to be the ruggedness of the resulted pattern. On the contrary, the " $3-5 \mathrm{pt}$ " method is stable and much accurate for the equation excluding the friction term and the "4-5 pt" method for any kind of equations. The fictitious waves in small scale are extraordinarily small by these methods. The measures whereby one can expect the occurrence of the error are presented. They are derived from the difference between the differential and the difference equations, and they are related to the degree of the spatial undulation of the quantity concerned. Finally, using the techniques discussed, some sample calculations of 500 hour forecast are performed with respect to the patterns moving on a one-dimensional ringwise grid, and the numerical solutions are compared with the analytical solutions.
\end{abstract}

\section{Introduction}

The result of the numerical weather prediction based on the hemispherical barotropic model seems at present hardly available after the forecast time 60 hours. It is a problem whether the error comes from the numerical or from the physical reason. At any rate, according to the experience obtained at the Computation Centre in the Japan Meteorological Agency, the pattern predicted, say, at 40 hours shows much ruggedness. It needs, accordingly, the process of smoothing, and the pattern becomes flat as time goes on. Thus, it is readily inferred that in order to extend the forecast period beyond 48 hours there may exist a great difficulty in the numerical problem.

The present knowledge about the error pro- duced by the time integation of partial differential equation is very meagre, and it is almost impossible to know extent to which the computational results involve the numerical error.

As to the technique to solve the initial value problem under a given differential equation, there have hitherto been proposed, for instance, the method using the functional expansion series such as Fourier series, and Runge-Kutta-Gill method. It is true that the former may be effective for the linear equation which does not produce new small waves by interaction of two quantities, but it is quite inadequate for the equation with variable coefficient, and especially, for the non-linear equation. That is because the components analyzed from the initial pattern is compelled to include errors arising from taking the finite 
space difference. "Finite" is indeed an inevitable restriction imposed to us in practice. As Richtmyer stated (1957), since the function given by the Fourier series is relevant only at the net points, there is a great deal of arbitrariness in the choice of the coefficients. In other words, the components are the artificial products of the technique, though the composed pattern has, of course, a physical significance. Runge-Kutta-Gill method is a distinguished technique, but to the partial differential and the non-linear equation, it is too complicated to apply it. Furthermore, this method is designed only for the purpose of time extrapolation. The most essential obstacle for solving the partial differential equation, however, seems to lie in a slightly different point from it. After all, the appropriate method has not yet been found except the conventional method currently employed in the field of the numerical weather prediction.

In this paper, we first study the socalled "inherited error" in solving the differential equation numerically by the marching process. It is the trunction error produced by the approximation of the differential equation with the finite difference and is of an accumulative nature with increasing time step. Then, based upon the information thus obtained, a stable and accurate technique is presented and the method to ascertain the degree of accuracy is discussed. The fundamental principle we bear in mind is that, since the equations dealt with are usually much complicated, the method should be simple and be generally applicable without knowing the physical nature of the terms in the equation.

We shall describle the outline of the following sections in more detail. For the first place, the discussion is confined only to the method of time extrapolation, and the 3-level and the 4-level methods are proposed, where the numbers indicate those of the consecutive time steps used for extrapolation. Further, it is described that there can hardly exist the computationally stable methods using the time levels more than 5, where the scheme is restricted to be "explicit", i.e., only one future time step is contained in the scheme. Second, the above methods are applied to a linear equation involving the advection term where the advecting flow is spatially constant, and further, to an equation involving both the advection and the frictional terms. As is generally known, the frictional term needs a slightly special consideration in solving it numerically by the usual centered difference method. It is shown, however, that the 4 level method mentioned above does not require such a consideration but is generally applicable to any type of equation. Next, the approximate evaluation of the error is made, using the remainder of the difference equation.

Third, we extend the discussion to the case where the advecting speed is variable with space. It is characteristic in this case that the interaction of the advecting flow and the advected quantity gives arise new waves. These waves become the cause of ruggedness of the pattern and turn out to be a difficulty for computation. The error in this case increases with time step, but does not belong to that due to the computational instability generally known. The rate of its increase is a function of the scale of wavelength. Speaking it in the terms of the finite difference computation, it is related to the number of gridpoints included in one wave. But the above statement is still incomplete, because it is impossible to define a wave length in the complicated pattern without making resourse to the Fourier analysis. As described before, however, because the components analyzed by the Fourier analysis are the artificial products, it is desired to get a measure substituting the concept of the wave, or a measure by which one can expect the rate of the occurrence of error. In this meaning, we propose a quantity consisting of the higher finite space difference of the value we are treating. We next assert that the error arising from the approximation of the space derivative is fairly large beyond our expectation, and accordingly it is very effective to reduce it as much as possible. We tested the 5-point space difference method and found that it gives a much accurate and stable result compared with the 3 point method conventionally used. Finally, some examples of 500 hour forecast are shown based on the informations and techniques obtained in this study. 


\section{Various methods of time extrapolation}

\section{(a) Preliminary notes}

First, let us consider to solve the following equation numerically

$$
\frac{d \varphi}{d t}=F(\varphi) .
$$

It is usual to adopt the analogue to $d \varphi / d t$ as follows

$$
\begin{aligned}
\left(\varphi_{\tau+1}-\varphi_{\tau}\right) / \Delta t & =\frac{d \varphi}{d t}+\text { Error, } \quad(2 \text { level }) \\
\text { Error } & =\frac{1}{2} \Delta t \cdot \frac{d^{2} \varphi}{d t^{2}}
\end{aligned}
$$

or

$$
\begin{aligned}
\left(\varphi_{\tau+1}-\varphi_{\tau-1}\right) / 2 \Delta t & =\frac{d \varphi}{d t}+\text { Error, } \quad(3 \text { level }) \\
\text { Error } & =\frac{1}{12} \Delta t^{2} \frac{d^{3} \varphi}{d t^{3}}
\end{aligned}
$$

where $\varphi_{\tau}$ denotes $\varphi$ at the time step $\tau$. Usually the former scheme is called the "forward difference" method and the latter the "centered difference" method. For the sake of convenience, however, we term them the " 2 level" and the " 3 level" methods (implicitly involving $\varphi_{\tau}$ ), respectively, according to the number of time steps of $\varphi$ used.

Now we look at the errors associated to the above two methods. It is seen that the former is of the order of $\Delta t$ and the latter of $\Delta t^{2}$, and therefore, the latter scheme seems to give less error than the former.

Next, in order to raise up the accuracy of approximation, we extend these schemes to the 4 level system. For this purpose, the Taylor series expansions of $\varphi_{\tau+1}, \varphi_{\tau-1}$ and $\varphi_{\tau-2}$ will be written

$$
\begin{aligned}
\varphi_{\tau+1}= & \varphi_{\tau}+\Delta t \frac{d \varphi}{d t}+\frac{1}{2 !} \Delta t^{2} \frac{d^{2} \varphi}{d t^{2}}+\frac{1}{3 !} \Delta t^{3} \frac{d^{3} \varphi}{d t^{3}}+\cdots \cdots \\
\varphi_{\tau-1}= & \varphi_{\tau}-\Delta t \frac{d \varphi}{d t}+\frac{1}{2 !} \Delta t^{2} \frac{d^{2} \varphi}{d t^{2}}-\frac{1}{3 !} \Delta t^{3} \frac{d^{3} \varphi}{d t^{3}}+\cdots \cdots \\
\varphi_{\tau-2}= & \varphi_{\tau}-2 \Delta t \frac{d \varphi}{d t}+\frac{1}{2 !}(2 \Delta t)^{2} \frac{d^{2} \varphi}{d t^{2}} \\
& -\frac{1}{3 !}(2 \Delta t)^{3} \frac{d^{3} \varphi}{d t^{3}}+\cdots \cdots
\end{aligned}
$$

Multiplying $\alpha, \beta$ and $\gamma$ to the first, the second and the third formulas, we have

$$
\begin{aligned}
& \alpha \varphi_{\tau+1}+\beta \varphi_{\tau-1}+\gamma \varphi_{\tau-2}-(\alpha+\beta+\gamma) \varphi_{\tau} \\
& =\Delta t \frac{d \varphi}{d t}(\alpha-\beta-2 \gamma)+\frac{1}{2 !} \Delta t^{2} \frac{d^{2} \varphi}{d t^{2}}\left(\alpha+\beta^{3}+4 \gamma\right) \\
& \quad+\frac{1}{3 !} \Delta t^{3} \frac{d^{3} \varphi}{d t^{3}}(\alpha-\beta-8 \gamma)
\end{aligned}
$$

$$
+\frac{1}{4 !} \Delta t^{4} \frac{d^{4} \varphi}{d t^{4}}(\alpha+\beta+16 \gamma)+\cdots \cdots
$$

There can be derived various schemes according to the values of $\alpha, \beta$ and $\gamma$. Among them, we first try to obtain the scheme giving the highest order of error. It is obvious in (2.4) that the coefficients satisfying the following relations, i.e.,

$$
\begin{aligned}
& \alpha+\beta+4 \gamma=0, \\
& \alpha-\beta-8 \gamma=0,
\end{aligned}
$$

will give the error of the order of $\Delta t^{3}$, if divided by $\Delta t$. Thus we have $\beta=-3 \alpha$ and $\gamma=\frac{1}{2} \alpha$, and therefore

$$
\begin{gathered}
\frac{1}{3}\left(\varphi_{\tau+1}-3 \varphi_{\tau-1}+\frac{1}{2} \varphi_{\tau-2}+\frac{3}{2} \varphi_{\tau}\right) / \Delta t \\
\fallingdotseq \frac{d \varphi}{d t}+\text { Error, } \quad(4 \text { level } \mathrm{A}) \\
\text { Error }=\frac{1}{12} \Delta t^{3} \frac{d^{4} \varphi}{d t^{4}} .
\end{gathered}
$$

This method will be termed the " 4 level A method".

\section{(b) Remarks on the test}

Before proceeding to the test of the above methods, we shall offer some remarks on the starting value in marching process, and on the property of the forcing function $F(\varphi)$ in eq. (2.1).

On the method of determination of the start-

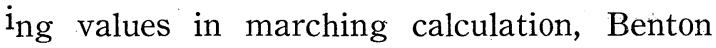
(1958), Hinkelmann (1959) and Arakawa (1960) assert that the time interval at the starting point should be half of that used in the further steps, for the forward method used in the first time step makes arise a considerable error. The practical scheme is shown in Fig. 2 and

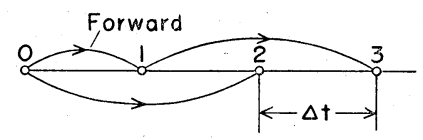

Fig. 1. Schematic illustration of the usual method for determination of the starting value. Abscissa gives time.

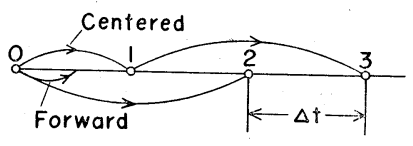

Fig. 2. Schematic illustration of the half time interval stepping method. 
will be called "the half time interval stepping method", while the conventional method is shown in Fig. 1. These circumstances are considered in the following way. The centered difference scheme produces two kinds of mode of pattern; one is physical and the other computational (Platzman 1954, Reiser 1957, Miyakoda 1959). Thus in calculation of the marching problem (the initial value problem), there need two initial conditions because of the nature of the centered difference method. At that time, two conditions should be taken so as not to produce much computational mode. It is then presumed that the half stepping method gives the condition suitable for this purpose. The practical result will be shown later.

Secondly, on the property of forcing function in eq. (2.1), Miyakoda (1959) claims that if we assume the functional form of $F(\varphi)$ as

$$
F(\varphi)=-\alpha \cdot \varphi,
$$

then in the centered difference sheme i.e., the 3 level method, the numerical solution turns out to be absolutely stable, conditionally stable and absolutely unstable, according to the condition that $\alpha$ is negative, imaginary and positive, respectively. In other words, when the forcing function is of the restitutive nature, that is, $\alpha$ is negative, the computational mode of the solution obtained by the centered difference scheme has a tendency of growing up with time step, and when it is of advecting nature, that is, $\alpha$ is imaginary, the error increases or does not if or if not a certain condition is satisfied, where the criterion is usually called the computational stability condition.

The representative of the restitutive forcing function is the frictional term. In this sense, we shall take up this term in the later experiment.

Next, it should be noted that, for simplicity, we first confine the discussion to timedependent problem. Namely, the equation

$$
\frac{\partial \varphi}{\partial t}=-U \frac{\partial \varphi}{\partial x}+\nu \frac{\partial^{2} \varphi}{\partial x^{2}}
$$

is modified by insertion of

to

$$
\varphi=A(t) \sin m x+B(t) \cos m x
$$

$$
\frac{d A}{d t}=U m \cdot B-\nu m^{2} \cdot A
$$

$$
\frac{d B}{d t}=-U m \cdot A-\nu m^{2} \cdot B .
$$

These are the equations treated first, in which the first term corresponds to the advecting term (the phase of the solution wave changes) and the latter the restitutive.

\section{(c) Test}

On the items mentioned above, the tests were made using the equations of (2.8) and (2.9). The values adopted at the initial time were as follows

$$
\begin{aligned}
& A_{t=0}=1, \\
& B_{t=0}=0 .
\end{aligned}
$$

First, the analytical solution was obtained by (2.8) and (2.9), i.e.,

$$
\varphi(t)=A_{0} \cdot \sin m(x-U t) e^{-\nu t},
$$

or using the relation that $t=\tau \cdot \Delta t$, where $\Delta t$ is the time interval, we have

$$
\varphi(\tau)=A_{0} \cdot \sin m(x-U \tau \Delta t) e^{-\nu \tau \Delta t} .
$$

On the other hand, the numerical solutions of $A(\tau)$ and $B(\tau)$ were calculated by the respective finite difference methods, and the following quantities were calculated, i.e.,

$$
\begin{aligned}
& A_{c}(\tau)=\sqrt{A(\tau)^{2}+B(\tau)^{2}}, \\
& U_{c}(\tau)=\tan ^{-1}[B(\tau) / A(\tau)] / m \Delta t .
\end{aligned}
$$

These quantities are the amplitude and the phase speed in the numerical solution. Then, the deviations of amplitude and phase were calculated by

$$
\begin{aligned}
\Delta A & =A_{0}-A_{c}(\tau), \\
\text { Phase lag } & =\left[U-U_{c}(\tau)\right] \cdot t .
\end{aligned}
$$

In the tests hereafter, the two quantities above were taken as the indication of accuracy.

Under the condition that $U m \Delta t=0.25, \nu m^{2} \Delta t$ $=0$, the methods to determine the starting values were compared (Figs. 3 and 4), where $\Delta t=1$ hour and $U=10 \mathrm{~m} / \mathrm{sec}$. Fig. 3 was obtained by the usual stepping method (Fig. 2), and Fig. 4 by the half time interval stepping method. As is seen in these figures, the usual method produces a considerable discrepancy which is inconsistent to the centered difference method, and the error produced at the beginning continues forever in the further steps. Thus, it is clear that the half time interval stepping method is favourable, and in all the treatment hereafter, this method will be utilized. 


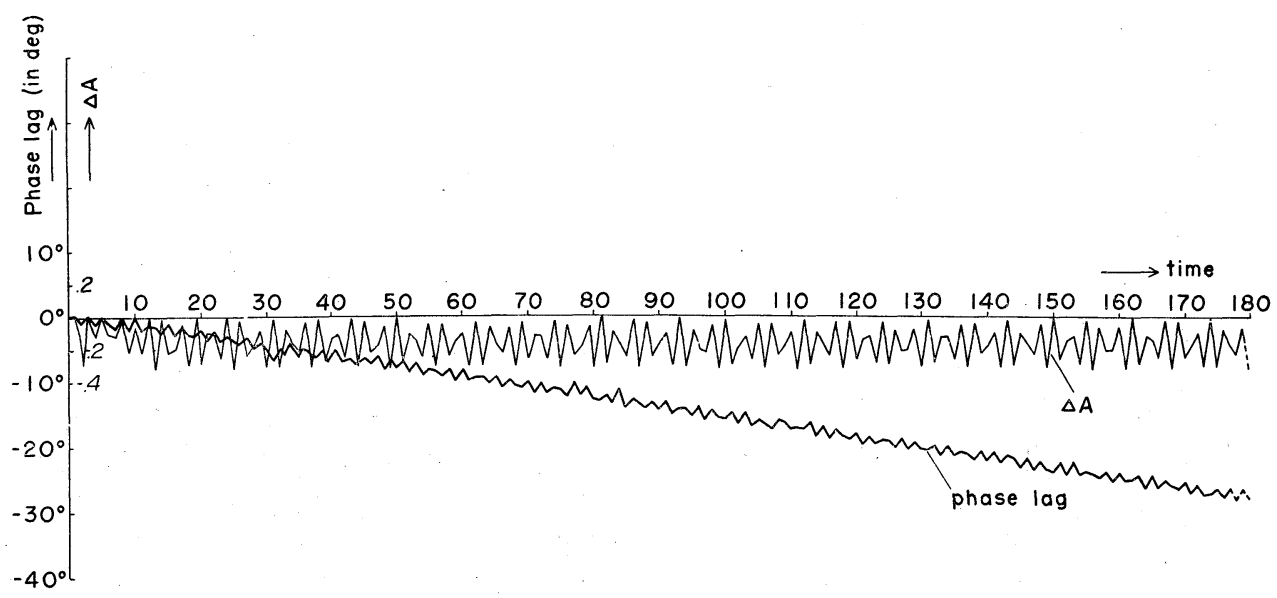

Fig. 3. Time variation of errors of amplitude $\Delta A$ and of phase lag in unit of degree. The determination of starting value is by the usual method shown in Fig. 1, the time extrapolation is made by the 3 level method and $m U \Delta t=0.25$. Abscissa gives time in unit of hour.

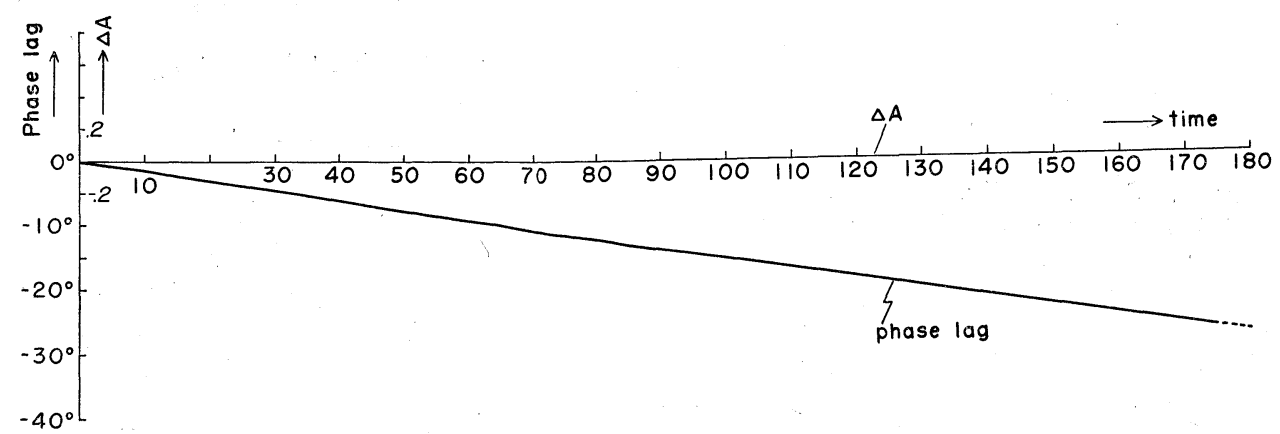

Fig. 4. Same as Fig. 3, but the determination of the starting value is made by the half time interval stepping method shown in Fig. 2.

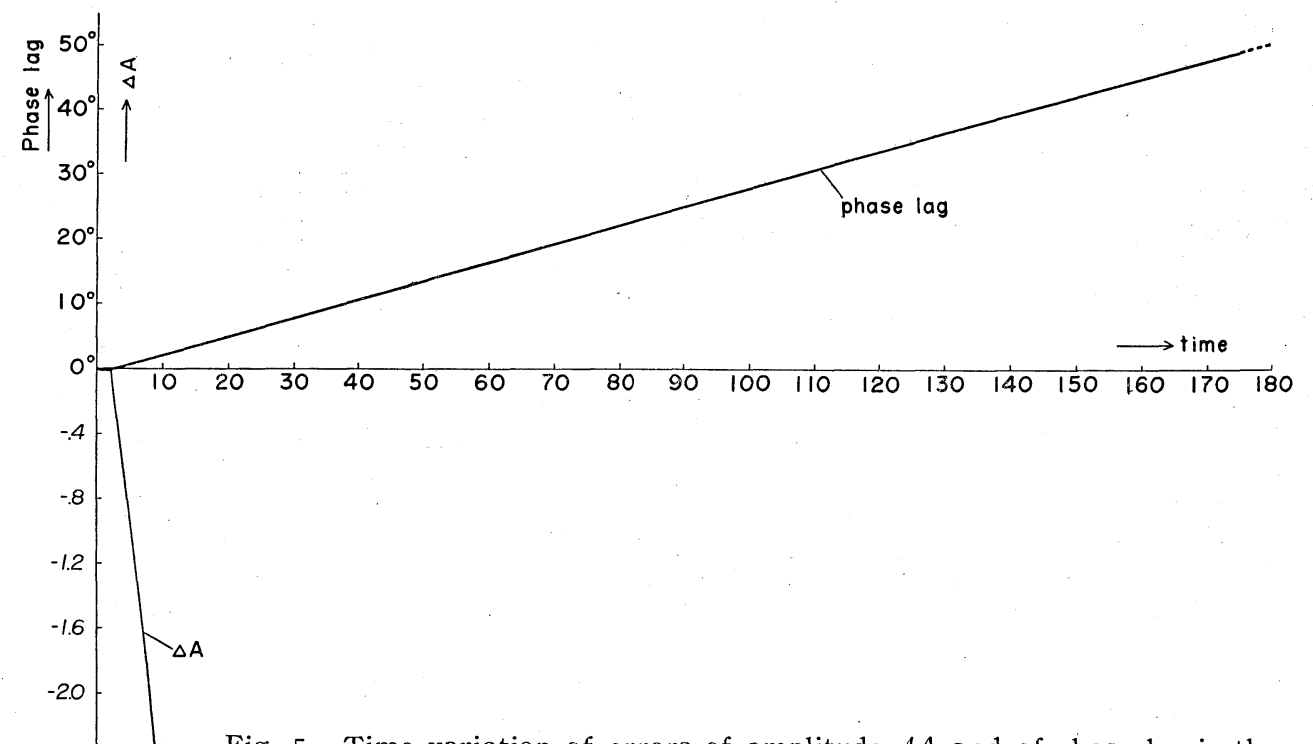

Fig. 5. Time variation of errors of amplitude $\Delta A$ and of phase lag in the unit of degree, by the " 2 level" method for time extrapolation. 


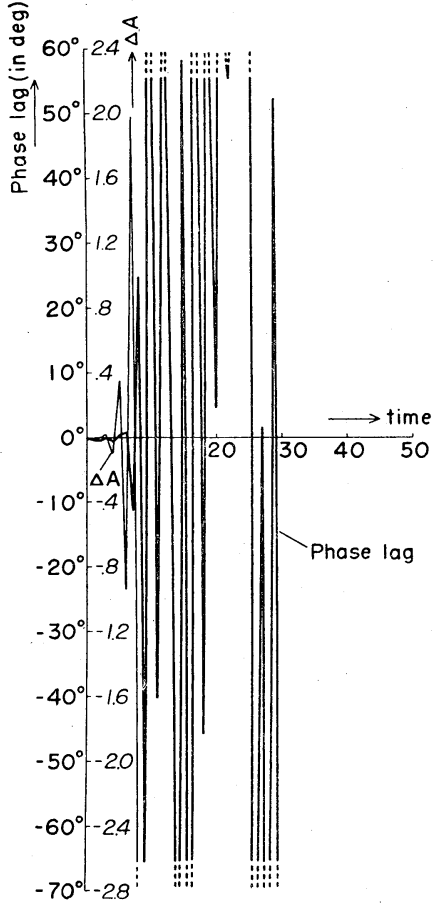

Fig. 6. Same as Fig. 5 but by the " 4 level A" method for time extrapolation.

Secondly, the extrapolation schemes were compared; the methods taken up were the 2 level and the 4 level $\mathrm{A}$, and the values employed were the same as those of the above test. The results are shown in Figs. 5 and 6. It is seen that the 2 level method gives a rapidly and monotonically growing error, the 3 level is fairly satisfactory (Fig. 4) and the 4 level $\mathrm{A}$ is absolutely unstable.

The important conclusion obtained above is that, as seen in the result of the 4 level A, the superiority of the scheme can not be judged only from the order of errors in the Taylor series expansion. The order of error indicates the accuracy of only the physical mode of the finite difference solution, but it does not give any information of the computational mode and stability. Generally there exist two requirements for the scheme, i.e., the stability and the accuracy. They are often incompatible; if we want to raise up the accuracy, then the stability is lost.

(d) The other extrapolation methods

In the case of the 4 level $\mathrm{A}$, the oscillation

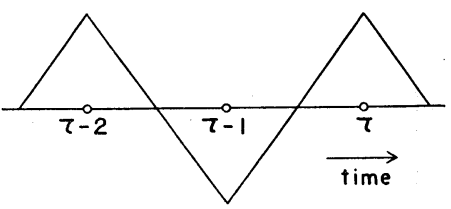

Fig. 7. Schematic illustration of the variation of error with increasing time step.

of the period of 2 time steps grows rapidly in a short time (Fig. 7). In order to suppress this kind of oscillation, the averaging process of the value $\varphi$ at three consecutive steps seems to be effective. In the centered difference scheme, making use of simple averaging, and putting

$$
\left[\left(\varphi_{\tau}+\varphi_{\tau-2}\right) / 2+\varphi_{\tau-1}\right] / 2
$$

instead of $\varphi_{\tau-1}$ in eq. (2.3), we have

$$
\begin{gathered}
{\left[\varphi_{\tau+1}-\frac{1}{4}\left(\varphi_{\tau}+2 \varphi_{\tau-1}+\varphi_{\tau-2}\right)\right] / 2 \Delta t} \\
=\frac{d \varphi}{d t}+\text { Error, }(4 \text { level B }) \\
\text { Error }=\frac{1}{8} \Delta t \frac{d^{2} \varphi}{d t^{2}} .
\end{gathered}
$$

This scheme was designed by the Working Group for the numerical weather prediction in Tokyo, 1958.

The error produced is much larger than that of the 3 level method. The above method will be called the " 4 level B" method.

On the other hand, another stable and accurate method in 4 level system is obtained by setting the conditions for (2.6) as follows instead of (2.5)

$$
\begin{array}{r}
\alpha+\beta+4 \gamma=0, \\
\alpha+2 \beta=0 .
\end{array}
$$

The latter condition implies that the errors distributed as $+\varepsilon,-\varepsilon,+\varepsilon$ at the time steps $\tau, \tau-1, \tau-2$, respectively, shall be damped (Fig. 7).

Thus, we have $\beta=-\alpha / 2, \gamma=-\alpha / 8$ and

$$
\begin{gathered}
{\left[\varphi_{\tau+1}-\frac{1}{8}\left(3 \varphi_{\tau}+4 \varphi_{\tau-1}+\varphi_{\tau-\bar{z}}\right)\right] 4 / 7 \Delta t} \\
=\frac{d \varphi}{d t}+\text { Error, } \quad(4 \text { level C) } \\
\text { Error }=\frac{5}{21} \Delta t^{2} \frac{d^{3} \varphi}{d t^{3}} .
\end{gathered}
$$

This scheme will be called the " 4 level C" method. The accuracy of the method of 4 


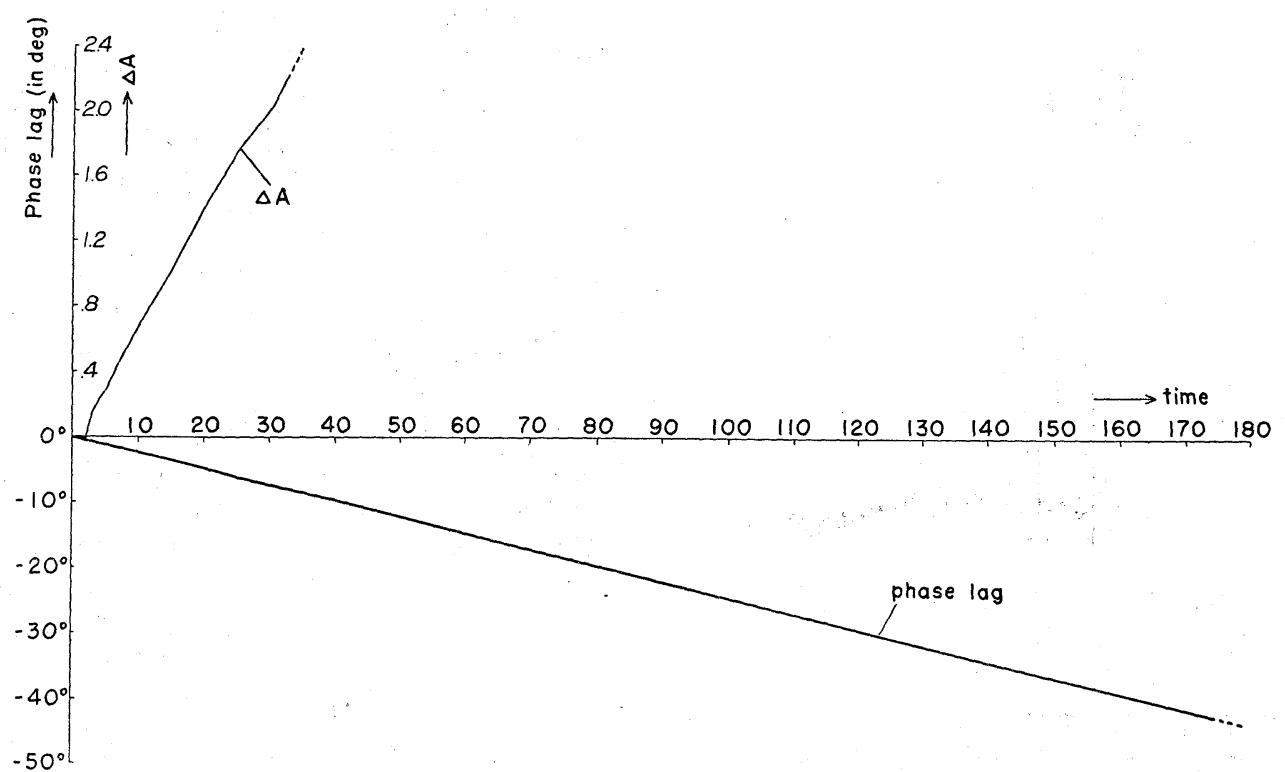

Fig. 8. Same as Fig. 5 but by the " 4 level B" method for time extrapolation.

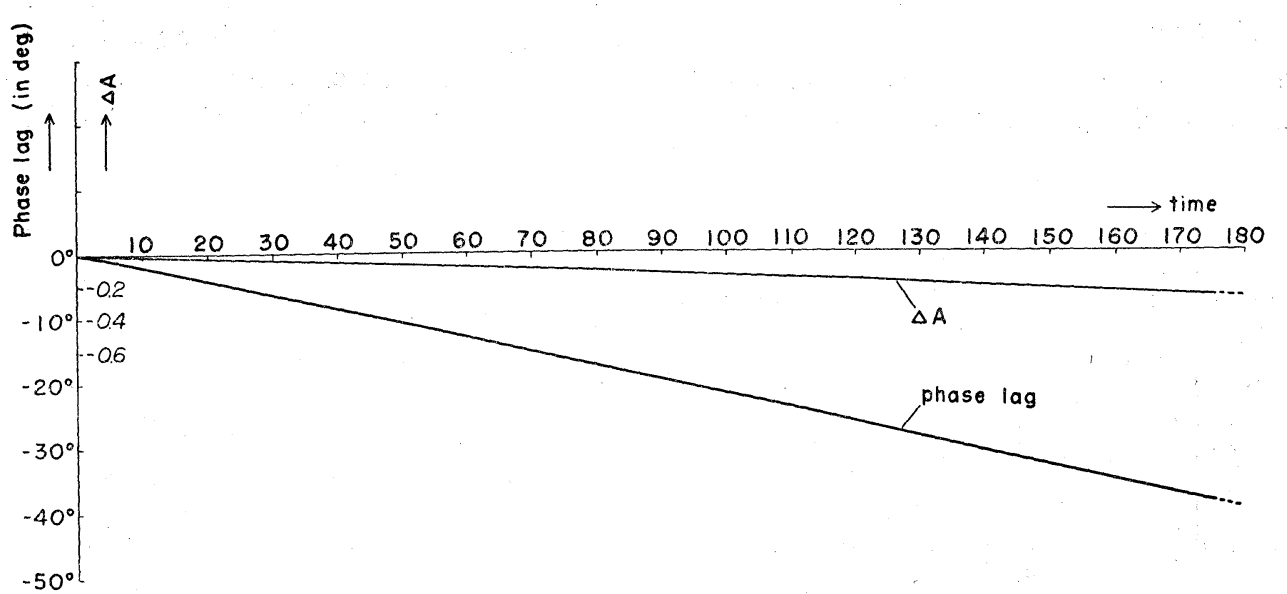

Fig. 9. Same as Fig. 5 but by the " 4 level C" method for time extrapolation.

level $\mathrm{C}$ seems to be a little larger than that of 3 level, but smaller than that of 4 level B.

The tests were made on the methods of 4 level $\mathrm{B}$ and 4 level $\mathrm{C}$ under the same condition as before (Figs. 8 and 9). As was expected, these methods are completely stable, and the deviations are larger in the 4 level $\mathrm{B}$ than in the 3 level and the 4 level $\mathrm{C}$.

In order to get a higher accuracy, we further extend the system to 5 and 6 levels. For instance, as the 6 level method in which the alternating oscillation with time step is designed to be damped, we have

$$
\frac{32}{93}\left[\varphi_{\tau+1}-\frac{1}{128}\left(-135 \varphi_{\tau}+208 \varphi_{\tau-1}\right.\right.
$$

$$
\begin{gathered}
\left.\left.+164 \varphi_{\tau-2}-144 \varphi_{\tau-3}+35 \varphi_{\tau-4}\right)\right] / \Delta t \\
=\frac{d \varphi}{d t}+\text { Error, } \quad(6 \text { level }) \\
\text { Error }=\frac{67}{465} \Delta t^{4} \frac{d^{5} \varphi}{d t^{5}} .
\end{gathered}
$$

But the result (Fig. 10) shows that this method is utterly unstable. It is probable that the oscillation of the period different from that of three time steps would occur. In order to get a stable method for any kind of oscillations, the values of coefficients appearing in the scheme should be in the same sign except that for $\varphi_{\tau+1}$. But it is impossible to establish a scheme satisfying this condition. 


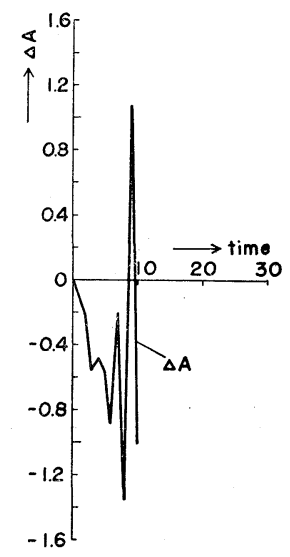

Fig. 10. Same as Fig. 5 but by the " 6 level" method for time extrapolation.

Therefore, we confine the scheme tested hereafter to the 3 level and the 4 level $\mathrm{C}$ methods.

\section{(e) Test on the restitutive term}

The applicability for the restitutive term is examined with respect to the schemes of 3 level and 4 level $C$. The values used are $U m \Delta t=0.25$ and $\nu m^{2} \Delta t=0.025$. As is expected, the 3 level method is unstable for this term even if the half time interval stepping method is adopted (Fig. 11). Exactly speaking, if it were possible to eliminate the computational mode completely by adopting a suitable starting method, the oscillation as in Fig. 11 would not be induced. Practically, however, it is impossible. On the other hand, the 4-level $\mathrm{C}$ method gives a stable solution (Fig. 11) though the monotonically increasing error is produced.

So far as the treatment of the frictional term is concerned, there have been proposed several methods which give a stable solution; for instance, the "implicit" methods (Richtmeyer 1957) and the "smoothing" method (Miyakoda 1959). The merit of the 4-level $\mathrm{C}$ method is that there needs no special consideration for the respective term to be treated, and the method is generally applicable. In the case of a simple linear equation, the classification of the term mentioned before

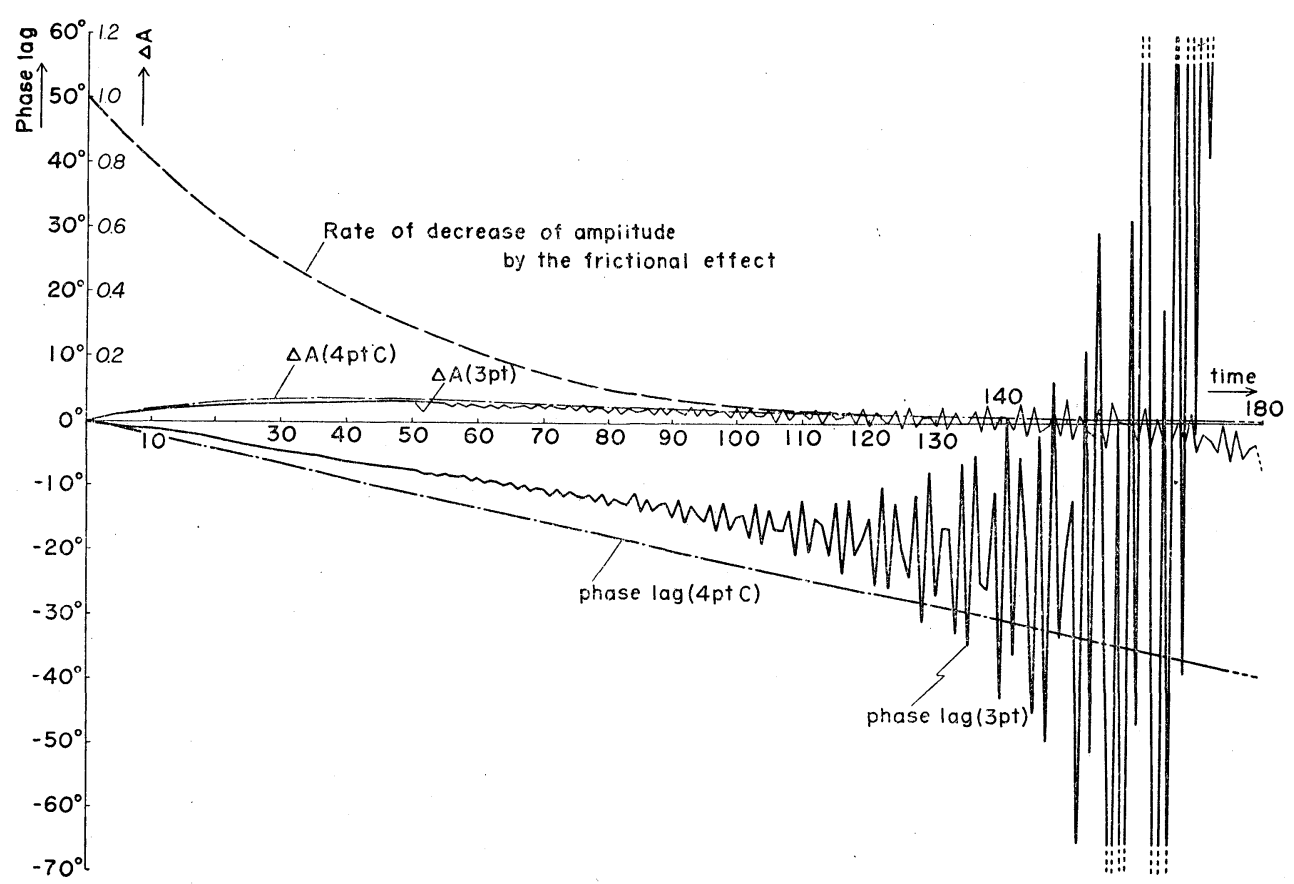

Fig. 11. Time variation of errors of amplitude $\Delta A$ and phase lag for an equation involving the frictional term. The values adopted are $m U . \Delta t=0.25$ and $m^{2} \nu . \Delta t=0.025$. One set of curves is obtained by the " 3 level" method, which shows the erroneous oscillation, and the other by the " 4 level $\mathrm{C}$ " method. The rate of decrease of amplitude due to the friction effect is shown, for reference, by the dashed line. 
is possible, but not in the case of the complicated system of the equations. In such a case, the 4 level $\mathrm{C}$ method may be especially effective.

\section{(f) Conclusions}

We studied the time extrapolation methods, restricting it to the explicit schemes. That is because other methods, for instance, the implicit method, or the method using the "predictor" and the "corrector" are somewhat complicated for treatment and besides they require a lot of time for computation.

After the tests of methods, we get the conclusions as follows.

(1) The 2-level method (the forward method) produces too much error.

(2) The 3-level method is, to some extent, accurate and stable for computing the equation consisting of the advecting or the amplifying terms, but it is unstable for the restitutive term.

(3) The 4 level $\mathrm{B}$ and the 4 level $\mathrm{C}$ methods are stable for any type of equation. But the former produces much error, and the error in the latter scheme is almost comparable with that of the 3 level method.

(4) The 4 level $\mathrm{A}$ and the 6 level methods are utterly unstable for any type of equation.

(5) Thus, we presume that there can hardly exist a stable method for extrapolation using the time steps more than 5 .

(6) We would like to recommend to use the 3 level method for a certain class of equation and the 4 level $\mathrm{C}$ method for any type of equation, though the latter scheme is slightly complicated in treatment.

\section{The advection equation of constant coefficient}

In treating the time-space dependent partial differential equation, the stability and the accuracy of the schemes should be considered from both points of view of space and time. Really, the error arising from the analoguing the time derivative sometimes cancels that from the space derivative. These circumstances together with the method of estimation of error and the problem associated with it shall be described in the following.

(a) The equation treated and the space difference
The differential equation dealt with hereafter will be prescribed, i.e.,

$$
\frac{\partial \varphi}{\partial t}=-U \frac{\partial \varphi}{\partial x}
$$

where $U$ is assumed constant with time and space. Then, we consider the case that the initial condition is as follows

$$
\text { at } t=0, \varphi=A_{0} \sin m x .
$$

In order to solve the above equation, the right hand side of the eq. (3.1) is approximated by the centered difference as follows

$$
\left(\frac{\partial \varphi}{\partial x}\right)_{i} \Rightarrow\left(\varphi_{i+1}-\varphi_{i-1}\right) / 2 \Delta s
$$

where $\Delta s$ is the gridsize. For the sake of convenience, this scheme will be called the " 3 point space difference" method. The error associated with the scheme (3.3) appears in the phase speed and it contributes to decrease it. (Thompson 1955, Magata 1957)

\section{(b) Exact evaluation of the errors}

First we discuss the inherited error arising from the approximation of the differential with the difference equation. The scheme taken up is based upon the 3 level method for time extrapolation and 3 point method for the space difference, which shall be called the "3-3 pt" method, i.e.,

$$
\left(\varphi_{\tau+1} *-\varphi_{\tau-1}{ }^{*}\right) / 2 \Delta t=-U \cdot\left(\varphi_{i+1} *-\varphi_{i-1} *\right) / 2 \Delta s,
$$

where $\varphi^{*}$ is the solution of finite difference equation. The exact solution is readily obtained as

$$
\varphi^{*}(\tau, i)=A_{0} \sin (\theta \cdot i-\lambda \cdot \tau)
$$

where

$$
\begin{aligned}
& \theta=\frac{2 \pi}{P} l, \\
& \lambda=\sin ^{-1}(k \sin \theta), \\
& k=U \Delta t / \Delta s,
\end{aligned}
$$

$l$ being the wave number, $P$ the total number of gridpoints. It is noted that the above solution is one of two solutions of the physical and the computational modes, and the above belongs to the former. The phase speed $U^{*}$ expressed by (3.5) and (3.6) is given by

$$
U^{*}=\sin ^{-1}\left(k \sin \frac{2 \pi}{P} l\right) / \Delta t,
$$

where the exact speed is $U$. Thus, the phase lag after the time $t$ becomes 
Phase lag $=t U\left[1-\frac{P}{2 \pi l} \frac{1}{k} \sin ^{-1}\left(k \sin \frac{2 \pi l}{P}\right)\right]$.

When $k$ is very near to zero, (3.8) becomes approximately

$$
\text { Phase lag } \fallingdotseq t U \frac{1}{6}\left(\frac{2 \pi l}{P}\right)^{2},
$$

and when $k$ is near to unity,

Phase lag $\fallingdotseq t U\left[\frac{2 \pi l}{P}-\tan \left(\sin \frac{2 \pi}{P} l\right)\right](1-k)$.

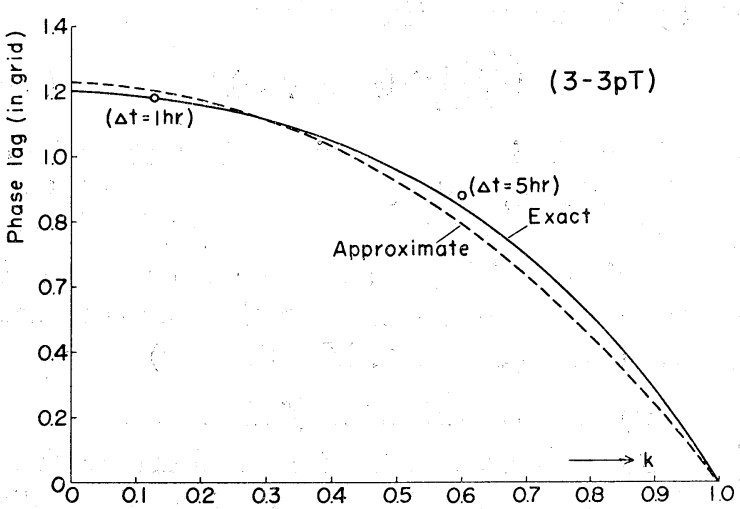

Fig. 12. Phase lag at the forecast time 100 hour against $k(=U \Delta t / \Delta s)$ in the $3-3 \mathrm{pt}$ scheme, where the solid line is the theoretical exact curve and the dashed the approximate curve. The plotted points are obtained by the practical numerical computation, and the figures in brackets indicate the time interval used in computation.

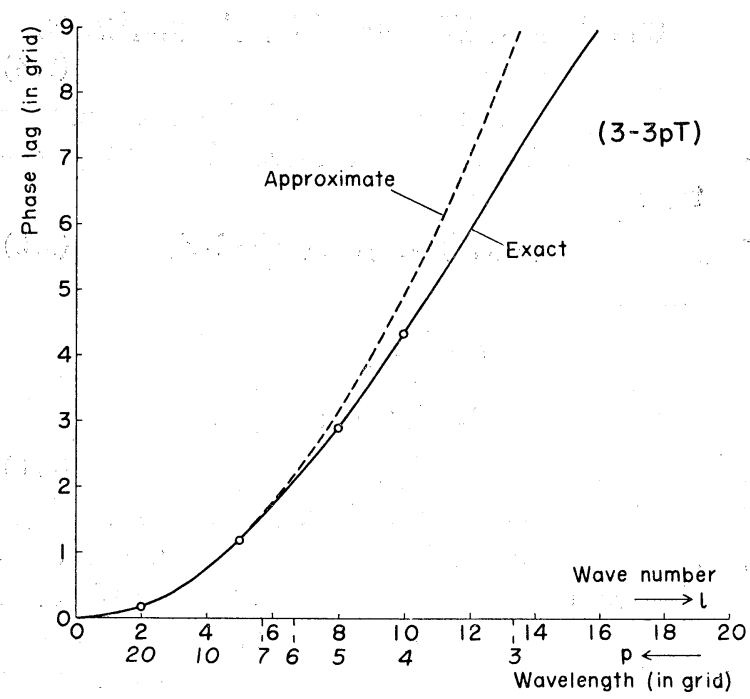

Fig. 13. Phase lag at the forecast time 100 hour against the wave number $l$ or wavelength $p$ in unit of gridpoint in the 3-3 pt scheme, where the solid line is the theoretical exact curve and the dashed the approximate curve. The plotted points are obtained by the practical numerical computation.
In Fig. 12, the phase lags at $t=100 \mathrm{hr}$ computed from eq. (3.8) are drawn against $k$, where $U=10 \mathrm{~m} / \mathrm{sec}, \Delta s=300 \mathrm{~km}, P=40$ grids, $l=5$ (the wavelength is 8 gridpts). Noted that when $\Delta t$ is small the error is large and that when $k=1$ the error becomes zero. But it should be remembered that $k=1$ is the limit from the view point of the computational stability. In Fig. 13, we show the phase lag against the wave number $l$ or the wavelength $\mathrm{i}_{\mathrm{n}}$ the unit of gridpoints, where $k=0$. It is clear that the smaller the wave length is, the larger the deviation becomes.

The conclusions obtained are that (1) even if we make small the time interval the deviation does never become small, and (2) when the wavelength is short the error becomes rapidly large.

\section{(c) Test}

Numerical test was carried out on the above problem, though it would be clear in advance that the theoretical values coincide with the numerical values. Speaking exactly, however, the following points are different in both treatments; (1) the scheme of determining the starting values and (2) the effect of rounding off. In the practical computation, we took 42 net-points and made the grid cyclic at the interval 40 gridpoints, i.e., $P=40$. The result shows that the agreement is almost complete (the plotted points in Figs. 12 and 13). Noted, however, that in the numerical calculation when $k$ becomes large near to unity, the solution fluctuates much violently.

The numerical solutions obtained at the gridpoints were analyzed into the Fourier series. As the result, we see that the amplitude given at the initial time does never change. Further, as is commonly conceived, the new wave does not occur in this system.

\section{(d) Approximate evaluation of errors}

Previously the exact estimation of the error was shown. But the analytical and the exact treatment is only possible in that simple case and for the 3 pt scheme. It is desired to obtain a general method to evaluate the error even in an approximate 
$f_{\text {orm. }}$.

As described before, the error for the $3 \mathrm{pt}$ method is given as follows; suppose the difference scheme be

$$
\varphi_{\tau+1}-\varphi_{\tau-1}=-k\left(\varphi_{i+1}-\varphi_{i-1}\right)+\varepsilon
$$

where $\varepsilon$ is the error of equation, then we have

$$
\begin{aligned}
\varepsilon= & 2 \Delta t\left(\frac{\partial \varphi}{\partial t}+U \frac{\partial \varphi}{\partial x}\right) \\
& +\frac{2}{3 !}\left(\frac{\partial^{3} \varphi}{\partial t^{3}} \Delta t^{3}+k \Delta s^{3} \frac{\partial^{3} \varphi}{\partial x^{3}}\right) .
\end{aligned}
$$

Hence, considering that

$$
\frac{\partial \varphi}{\partial t}+U \frac{\partial \varphi}{\partial x}=0
$$

the error becomes

$$
\varepsilon=\frac{2}{3 !} \Delta s^{2} \frac{\partial^{3} \varphi}{\partial x^{3}} k\left(1-k^{2}\right) .
$$

On the other hand, if we assume the numerical solution $\varphi^{*}$ as

$$
\varphi^{*}=A \sin m\left(x-c^{*} t\right)
$$

where $c^{*}$ is a numerical phase speed and it is in the relation with the actual speed $U$ as

$$
c^{*}=U+\delta U \text {. }
$$

Then, we get

$$
\begin{aligned}
& \varepsilon \doteqdot-2 \Delta t\left(\frac{\partial \varphi^{*}}{\partial t}+U \frac{\partial \varphi^{*}}{\partial x}\right) \\
& =2 \Delta t m \delta U A \cos m\left(x-c^{*} t\right) .
\end{aligned}
$$

If the expression (3:13) is inserted into (3.12), we have

$$
\varepsilon=\frac{1}{3} m^{3} \Delta s^{2} \Delta t U\left(1-k^{2}\right) A \cos m\left(x-c^{*} t\right) \text {. }
$$

The error expressed by (3.15) should be equal to that of (3.16). Therefore, we have

$$
\delta U \doteqdot-\frac{1}{3 !} m^{2} \Delta s^{2} U\left(1-k^{2}\right)
$$

or

$$
\text { Phase lag } \fallingdotseq \frac{1}{6} U t\left(\frac{2 \pi l}{P}\right)^{2}\left(1-k^{2}\right) \text {, }
$$

where the relation

$$
m=\frac{2 \pi l}{P \cdot \Delta s}
$$

is utilized.

The approximate formula of error (3.17) is much near to the exact relation (3.8). The values calculated by (3.17) are shown by the dashed lines in Figs. 12 and 13.

Since we see that the approximate method gives a satisfactory result, we shall next obtain the similar relation for the 4 level $\mathrm{C}$ method, i.e.,

$$
\therefore=\frac{2}{3 !} \frac{\partial^{3} \varphi}{\partial x^{3}} \Delta s^{3} k\left(1-\frac{10}{7} k^{2}\right),
$$

therefore

$$
\text { Phase lag } \div \frac{1}{6} U t\left(\frac{2 \pi l}{P}\right)^{2}\left(1-\frac{10}{7} k^{2}\right) .
$$

The curves of phase lag $-k$ and of phase lag $-l$ (or the wavelength) for the " $4-3 \mathrm{pt}$ method"

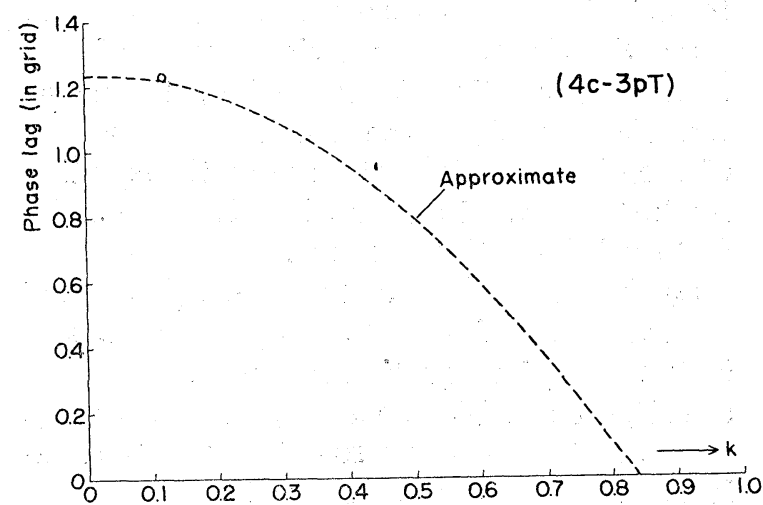

Fig. 14. Phase lag at the forecast time 100 hour against $k(=U \Delta t / \Delta s)$ in the $4 \mathrm{C}-3 \mathrm{pt}$ scheme, where the dashed line is the theoretical approximate curve. The plotted point is obtained by the practical numerical computation.

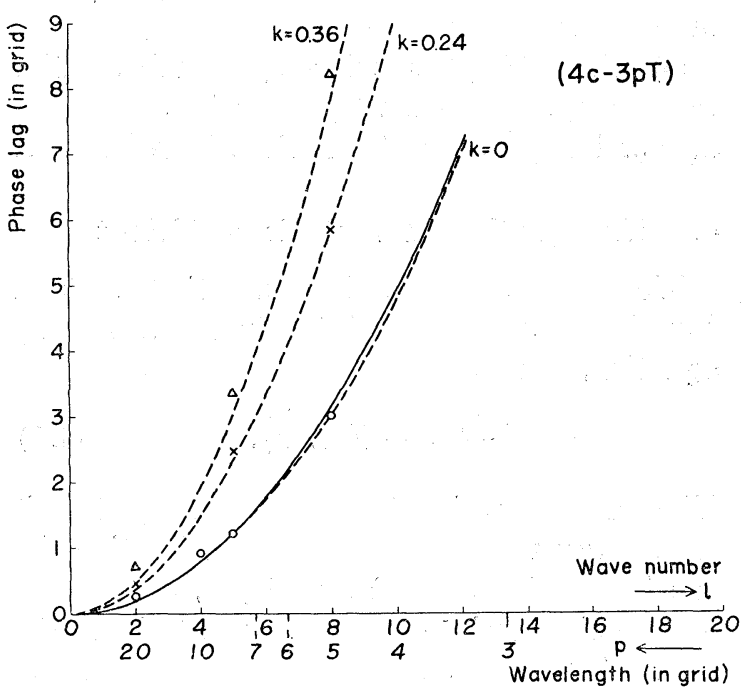

Fig. 15. Phase lag at the forecast time 100 hour against the wave number $l$ or the wavelength $p$ in unit of gridpoint in the $4 \mathrm{C}-3 \mathrm{pt}$ scheme. The dashed lines are the theoretical approximate curves with the parameter of $k(=U \Delta t / \Delta s)$, and the solid line which is the theoretical approximate curve of $k=0$ in the $3-3$ pt scheme is shown for comparison. The plotted points are obtained by the practical numerical computation, where the points $\bigcirc, \times$ and $\triangle$ belong to the cases of $k=0,0.24$ and 0.36 , respectively. 
are shown in Figs. 14 and 15, respectively, where the values obtained in the test are plotted. The agreement is satisfactory.

\section{(e) Conclusions}

The main purpose of the study in this section is to establish the method to evaluate the numerical error even in the approximate form. The conclusions obtained are as follows.

(1) So far as the physical mode of the numerical solution is concerned, the error formula derived as the dominant remainder of the difference from the differential equation by the Taylor series expansion gives the satisfactory information about it.

(2) In the scheme of the "3-3 pt" method with respect to time and space difference, and in the advection equation where the advecting speed $U$ is spatially constant, the phase lag of the numerical wave solution from the analytical solution is given by (3.17).

(3) In the scheme of the " $4 \mathrm{C}-3 \mathrm{pt}$ " method, the phase lag is given by (3.20).

(4) From the formulas shown above, we see that the phase lag is a function of $l / P$, which is the inverse of the number of gridpoints involved in one wavelength.

(5) Since $U \Delta t / \Delta s$ should be generally smaller than unity, the phase lag may be said to be almost independent of the time increment $\Delta t$.

\section{The equation of variable coefficient}

We now proceed to the case that $U$ is a function of space coordinate in the advecting equation, that is

$$
\frac{\partial \varphi}{\partial t}=-U(x) \frac{\partial \varphi}{\partial x} .
$$

The characteristic of this type of equation is that many waves are produced with time by the interaction of $U(x)$ and $\partial \varphi / \partial x$, even when a monochromatic wave is given at the initial time. Therefore, the errors are expected to appear not only in the phase speeds but also in the amplitudes.

(a) Outline of the problem and the treatment

It is a complicated thing to obtain the exact analytical solution of $\varphi$ in the eq. (4.1). Thus, we consider instead of (4.1) the following equation, i.e.,

$$
\frac{\partial \varphi}{\partial t}=-U(x) \frac{\partial \varphi}{\partial x}+f(x, t) .
$$

Now if we assume the functional forms of $\varphi$ and $U$ as follows

$$
\begin{gathered}
\varphi=A_{0} \sin m(x-c t), \\
U=U_{0}+U_{1} \sin n x,
\end{gathered}
$$

then the function of $f$ should be

$$
f=m U_{1} A_{0} \sin n x \cdot \cos m(x-c t) .
$$

Speaking inversely, if the equation is (4.2) and the functional forms of $U$ and $f$ are (4.4) and (4.5), then the solution of the equation must be (4.3). Of course, our interest is not focussed in obtaining a solution of the eq. (4.1), but in getting informations on the numerical error. Nevertheless, if the errors numerically produced from the term of $f$ are larger than or comparable with those from the advecting term $U(\partial \varphi / \partial x)$, one would encounter considerable difficulty to separate the errors. Fortunately to say, as will be described later, the latter is usually extraordinarily larger than the former. Thus, we shall hereafter adopt this way of attacking.

The tests were performed regarding the 3 pt method, and, for simplicity, one wave was assumed as the solution of $\varphi$, as mentioned above. The strategy we took was that the numerical solutions were obtained by the 3-3 pt method and, they were compared with the exact solutions. At the same time, the numerical solutions were analyzed into the Fourier series, and the amplitudes thus obtained were compared with the exact amplitudes.

(b) Test

The case taken up first was that

$$
\begin{array}{ll}
U_{0}=10 \mathrm{~m} / \mathrm{sec}, & U_{1}=3 \mathrm{~m} / \mathrm{sec}, \\
m=n=\frac{2 \pi l}{40 \cdot \Delta s}, & l=6, \\
A_{0}=1 & \\
\Delta t=1 \text { hour }, & \Delta s=300 \mathrm{~km} .
\end{array}
$$

The results are shown in Fig. 16, in which the profiles of the numerical and the exact solutions are compared every 20 hours. The most typical thing is that the numerical solutions come to deviate considerably from the exact solutions after 40 hours and that the small scale of waves are produced fictitiously. At the 

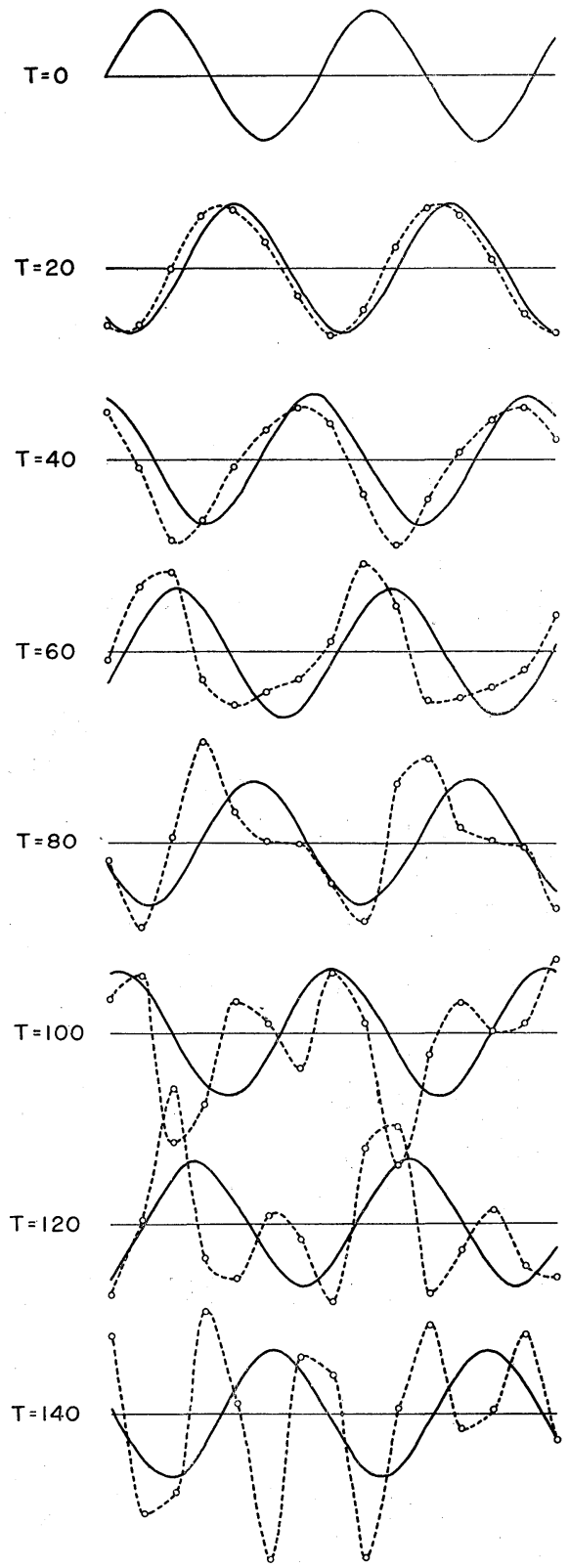

Fig. 16. Time evolution of the pattern at the interval of 20 hours, where the abscissas are $x$-coordinates. The solid lines are the exact solutions and the dashed the numerical solutions by the 3-3 pt method.

time of 120 hours, the numerical results are utterly different from the exact solution. It should be remarked that this erroneous ruggedness is not due to the so-called computational instability in the sense of Courant-
Friedrichs-Lewy's fashion, since the values of $U_{0} \Delta t / \Delta s$ and $U_{1} \Delta t / \Delta s$ are always fixed to be 0.120 and 0.036 , respectively. This errors come from the small waves erroneously produced by the interaction of $U$ and $\partial \varphi / \partial x$, which are both functions of space.

These features will be more apparent in Fig. 17, where the time evolution of the spectra of the numerical solutions analyzed by the Fourier analysis is shown. Of course, the exact solution in this case is a single line spectrum at the wave number 6 , and the spectra at the wave numer 0,12 and 18 are fictitiously produced. In Fig. 18, we illustrate how the errors of the wave number 0,6 and 12 develop witn time step. The increase of error at the small scale of wave is especially great.

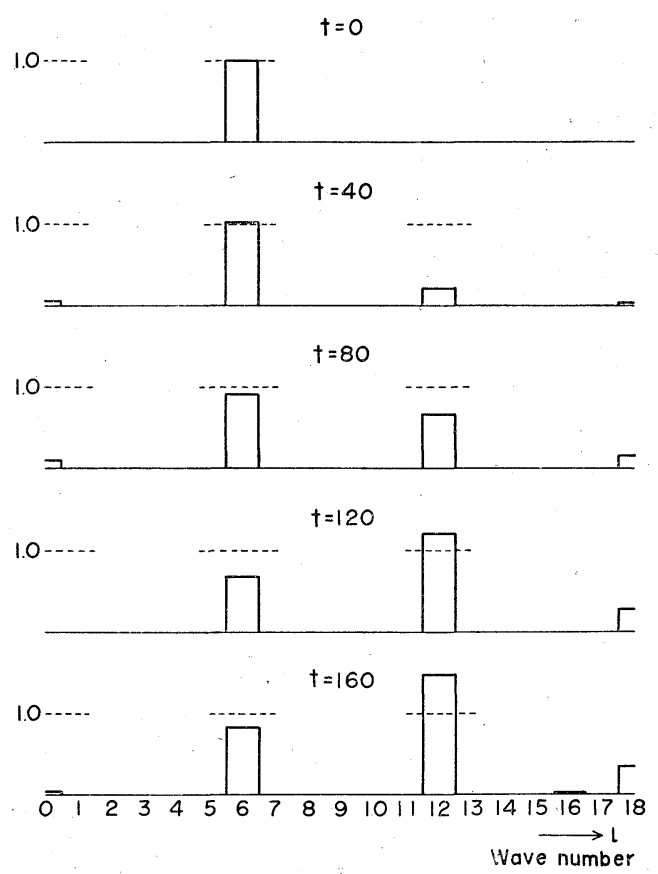

Fig. 17. Time evolution of the spectra of the numerical solutions shown in Fig. 16 at the interval of 40 hours. The exact solutions must be a unit spectrum at the wave number $l=6$.

As another test, we carried out the same calculation but with half the time increment $\Delta t$. The result is, however, not remarkably altered. 


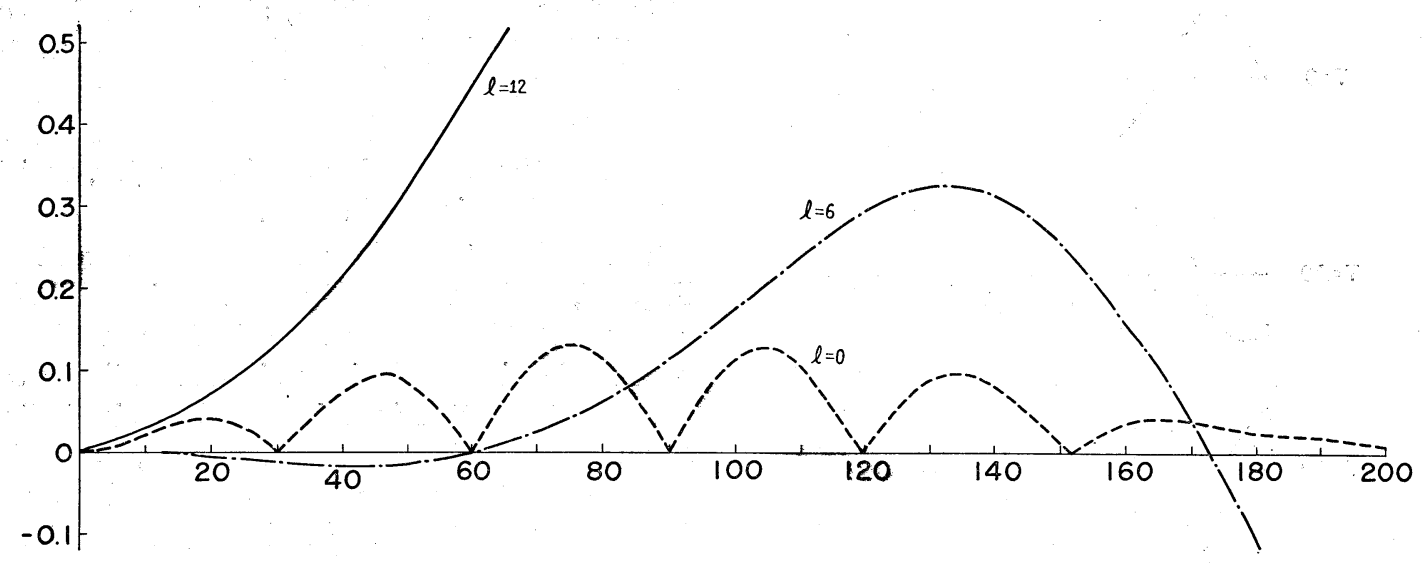

Fig. 18. Time variation of the deviations of amplitude in the Fourier components, where the amplitude of the significant wave is unity.

\section{(c) Theoretical consideration on error}

We now just consider theoretically the errors numerically produced by the 3-3 pt method. The numerical scheme we adopted is as follows.

$$
\begin{aligned}
& \left(\varphi_{\tau+1}{ }^{*}-\varphi_{\tau-1}{ }^{*}\right) / 2 \Delta t \\
& \quad=-U_{i}\left(\varphi_{i+1}{ }^{*}-\varphi_{i-1} *\right) / 2 \Delta s+f,
\end{aligned}
$$

where $\varphi^{*}$ is the solution of the finite difference equation.

Let us define $\varepsilon$ as the deviation of the numerical from the exact solution as follows

$$
\varphi=\varphi^{*}+\varepsilon \text {. }
$$

We insert the above relation into (4.2), and get

$$
\begin{aligned}
\varepsilon^{\prime} & =\frac{\partial \varepsilon}{\partial t}+U \frac{\partial \varepsilon}{\partial t} \\
& =-\left(\frac{\partial \varphi^{*}}{\partial t}+U \frac{\partial \varphi^{*}}{\partial x}-f\right) \\
& =-\left(\frac{\partial \varphi^{*}}{\partial t}+U \frac{\partial \varphi^{*}}{\partial x}-\frac{\partial \varphi}{\partial t}-U \frac{\partial \varphi}{\partial x}\right)
\end{aligned}
$$

where $\varepsilon^{\prime}$ is the error of the difference equation.

Here, for simplicity, we assume the special case, that

$$
\begin{aligned}
& \varphi=A_{0} \sin m\left(x-U_{0} t\right), \\
& U=U_{0}+U_{1} \sin m x .
\end{aligned}
$$

In order to express $\varepsilon^{\prime}$ by the wave functions, considering the wave components which will be produced by interaction of $U$ and $\varphi$, we put

$$
\begin{aligned}
\varphi^{*}= & D_{0}+D_{1} \sin m\left(x-c_{1} t\right) \\
& +D_{2} \sin 2 m\left(x-c_{2} t\right)+\cdots \cdots,
\end{aligned}
$$

where $D_{0}, D_{1}, D_{2}$ are the amplitudes of the solution of the wave number $0, m$ and $2 m$, respectively, and $c_{1}, c_{2}$ are the phase speeds of the solution of the finite difference equation. Then, the insertion of these functions into (4.9) leads to

$$
\begin{aligned}
\varepsilon^{\prime}= & {\left[\frac{d D_{0}}{d t}+\frac{1}{2} m U_{1}\left(D_{1} \sin m c_{1} t-A_{0} \sin m U_{0} t\right)\right] } \\
& +\left[m D_{1}\left(U_{0}-c_{1}\right)-m D_{2} U_{1} \sin m\left(c_{1}-2 c_{2}\right) t\right] \\
& \times \cos m\left(x-c_{1} t\right) \\
& +\left[\frac{d D_{1}}{d t}-m U_{1} D_{2} \cos m\left(c_{1}-2 c_{2}\right) t\right] \\
& \times \sin m\left(x-c_{1} t\right) \\
& +\left[\frac{d D_{2}}{d t}+\frac{1}{2} m U_{1} \cos m\left(2 c_{2}-c_{1}\right) t\right. \\
& \left.\times\left(D_{1}-A_{0} \cos m\left(c_{1}-c_{0}\right) t\right)\right] \sin 2 m\left(x-c_{2} t\right) .
\end{aligned}
$$

This relation indicates how the error of equation $\varepsilon^{\prime}$ is partitioned to the various elements and to the various components of the waves.

On the other hand, expanding the eq. (4.7) into the Taylor series, we get approximately

$$
\begin{gathered}
\frac{\partial \varphi^{*}}{\partial t}+U \frac{\partial \varphi^{*}}{\partial x}-f+\frac{1}{3 !} U \frac{\partial^{3} \varphi}{\partial x^{3}}\left(\Delta s^{2}-(U \Delta t)^{2}\right) \\
-\frac{1}{3 !} \Delta t^{2}\left(U^{2} \frac{\partial^{2} U}{\partial x^{2}}+U\left(\frac{\partial U}{\partial x}\right)^{2}\right) \frac{\partial \varphi^{*}}{\partial x} \\
-\frac{1}{2} \Delta t^{2} U^{2} \frac{\partial U}{\partial x} \frac{\partial^{2} \varphi^{*}}{\partial x^{2}}-\frac{1}{3 !} \Delta t^{2}\left(U \frac{\partial U}{\partial x} \frac{\partial f}{\partial x}\right. \\
\left.-U^{2} \frac{\partial^{2} f}{\partial x^{2}}+U \frac{\partial^{2} f}{\partial x \partial t}-\frac{\partial^{2} f}{\partial t^{2}}\right)+\cdots \cdots=0 .
\end{gathered}
$$

Using the newly defined quantities $\varepsilon_{1}, \varepsilon_{2}, \varepsilon_{3}$ and $\varepsilon_{4}$ the eq. (4.9) is expressed as follows

$$
\varepsilon^{\prime}=\varepsilon_{1}+\varepsilon_{2}+\varepsilon_{3}+\varepsilon_{4}
$$


and

$$
\begin{aligned}
\varepsilon_{1}= & \frac{1}{3 !} U \frac{\partial^{3} \varphi^{*}}{\partial x^{3}}\left(\Delta s^{2}-(U \Delta t)^{2}\right), \\
\varepsilon_{2}= & -\frac{1}{3 !} \Delta t^{2}\left(U^{2} \frac{\partial^{2} U}{\partial x^{2}}+U\left(\frac{\partial U}{\partial x}\right)^{2}\right) \frac{\partial \varphi^{*}}{\partial x}, \\
\varepsilon_{3}= & -\frac{1}{2} \Delta t^{2} U^{2} \frac{\partial U}{\partial x} \frac{\partial^{2} \varphi^{*}}{\partial x^{2}} \\
\varepsilon_{4}= & -\frac{1}{3 !} \Delta t^{2}\left(U \frac{\partial U}{\partial x} \frac{\partial f}{\partial x}-U^{2} \frac{\partial^{2} f}{\partial x^{2}}\right. \\
& \left.+U \frac{\partial^{2} f}{\partial x \partial t}-\frac{\partial^{2} f}{\partial t^{2}}\right) .
\end{aligned}
$$

The meaning of each term is as follows; $\varepsilon_{1}$ is the error due to the third space derivative of $\varphi^{*}$, that is, the skewness of the spatial undulation of $\varphi, \varepsilon_{2}$ and $\varepsilon_{3}$ are due to the first and second space derivatives of $U$ and $\varphi^{*}$, that is, the spatial undulation of the advecting flow, and $\varepsilon_{4}$ comes from the existence of the forcing term $f$. Inserting (4.11) into the above relations, we have, for instance,

$$
\begin{aligned}
\varepsilon_{1}= & \frac{1}{6} m^{3} \Delta s^{2}\left(-U_{0}+2 U_{0} U_{1}^{2}\left(\frac{\Delta t}{\Delta s}\right)^{2}+U_{0}^{3}\left(\frac{\Delta t}{\Delta s}\right)^{2}\right) \\
& \times D_{1} \cos m\left(x-c_{1} t\right) \\
& -\frac{1}{12} m^{3} \Delta s^{2}\left(U_{1}-U_{0}^{2} U_{1}\left(\frac{\Delta t}{\Delta s}\right)^{2}-\frac{1}{2} U_{1}^{3}\left(\frac{\Delta t}{\Delta s}\right)^{2}\right) \\
& \times D_{1} \sin m\left(x-c_{1} t\right) \\
& -\frac{1}{12} m^{3} \Delta s^{2}\left(U_{1}-7 U_{0}^{2} U_{1}\left(\frac{\Delta t}{\Delta s}\right)^{2}-2 U_{1}^{3}\left(\frac{\Delta t}{\Delta s}\right)^{2}\right) \\
& \times D_{1} \sin 2 m\left(x-\frac{1}{2} c_{1} t\right) .
\end{aligned}
$$

Now we introduce the following quantities

$$
k_{0}=U_{0} \Delta t / \Delta s, \quad k_{1}=U_{1} \Delta t / \Delta s,
$$

where these values are usually smaller than unity so that the computational stability condition is satisfied. Therefore, $k_{0}{ }^{3}, k_{0}{ }^{2} k_{1}$ are negligibly small compared with $k_{0}$. Thus, confining the discussion to the initial time, the eq. (4.17) is transformed to

$$
\begin{aligned}
\varepsilon_{1}= & -\frac{1}{6} \frac{(m \Delta s)^{3}}{\Delta t} k_{0} D_{1} \cos m x \\
& +\frac{1}{12} \frac{(m \Delta s)^{3}}{\Delta t} k_{1} D_{2} \sin m x \\
& -\frac{1}{12} \frac{(m \Delta s)^{3}}{\Delta t} k_{1} D_{1} \sin 2 m x \\
& +\cdots \cdots,
\end{aligned}
$$

and it is readily known that $\varepsilon_{2}, \varepsilon_{3}$ and $\varepsilon_{4}$ are small compared with $\varepsilon_{1}$, because $k_{0}$ and $k_{1}$ are smaller than unity.
Combining the eq. (4.12) and (4.18) and equating the coefficients for the same sinusoidal functions with space in both equations, we have

$$
\left(c_{1}-c_{0}\right) \Delta t / \Delta s=-\frac{1}{6}(m \Delta s)^{2} k_{0},
$$

and

$$
\begin{aligned}
& \frac{d D_{0}}{d t}=\frac{1}{12} \frac{(m \Delta s)^{3}}{\Delta t} k_{1} D_{1}, \\
& \frac{d D_{1}}{d t}=\frac{m \Delta s}{\Delta t} k_{1} D_{2}-\frac{1}{12} \frac{(m \Delta s)^{3}}{\Delta t} k_{1} D_{2}, \\
& \frac{d D_{2}}{d t}=-\frac{1}{2} \frac{m \Delta s}{\Delta t} k_{1}\left(D_{1}-A_{0}\right)+\frac{1}{12} \frac{(m \Delta s)^{3}}{\Delta t} k_{1} D_{1} .
\end{aligned}
$$

From these ralations it is seen that the phase lag of the numerical for the exact solution is almost the same as those shown in the previous section and the numerical amplitudes of the wave number of 0 and $2 m$ grows at the rate of $(1 / 12)\left((m \Delta s)^{3} / \Delta t\right) k_{1} D_{1}$ and that the wave of the wave number $m$ increases in proportion to $D_{2}$.

The present theoretical consideration does not result in complete success. It is, however, true that the error comes mainly from the term including $\partial^{3} \varphi / \partial x^{3}$ and that the error of wave number $2 m$ increases proportionally to $(m \Delta s)^{3} k_{1} D_{1} / \Delta t$.

\section{(d) Smoothing and filtering}

In order to suppress the occurrence of error at the small scale of wave, we made the tests on "smoothing" and "filtering".

The technique of "smoothing" was discussed in detail by Shuman (1955). Among them, we take the simplest scheme as follows

$$
\bar{\varphi}=\varphi+\frac{\alpha}{2}\left(\varphi_{i+1}+\varphi_{i-1}-2 \varphi_{i}\right),
$$

where the bar denotes the smoothing operation. In practice, we adopt $\alpha=0.016$ and the smoothing operations are applied every time step.

In Figs. 19 and 20, the results of the smoothing are shown for the same case as that we took up earlier. It is true that the supression of the small waves was, to some extent, successfully made, but the result is far from our satisfaction.

The filtering was tested with the same case. The procedure of this operation is as follows. 
$T=0$
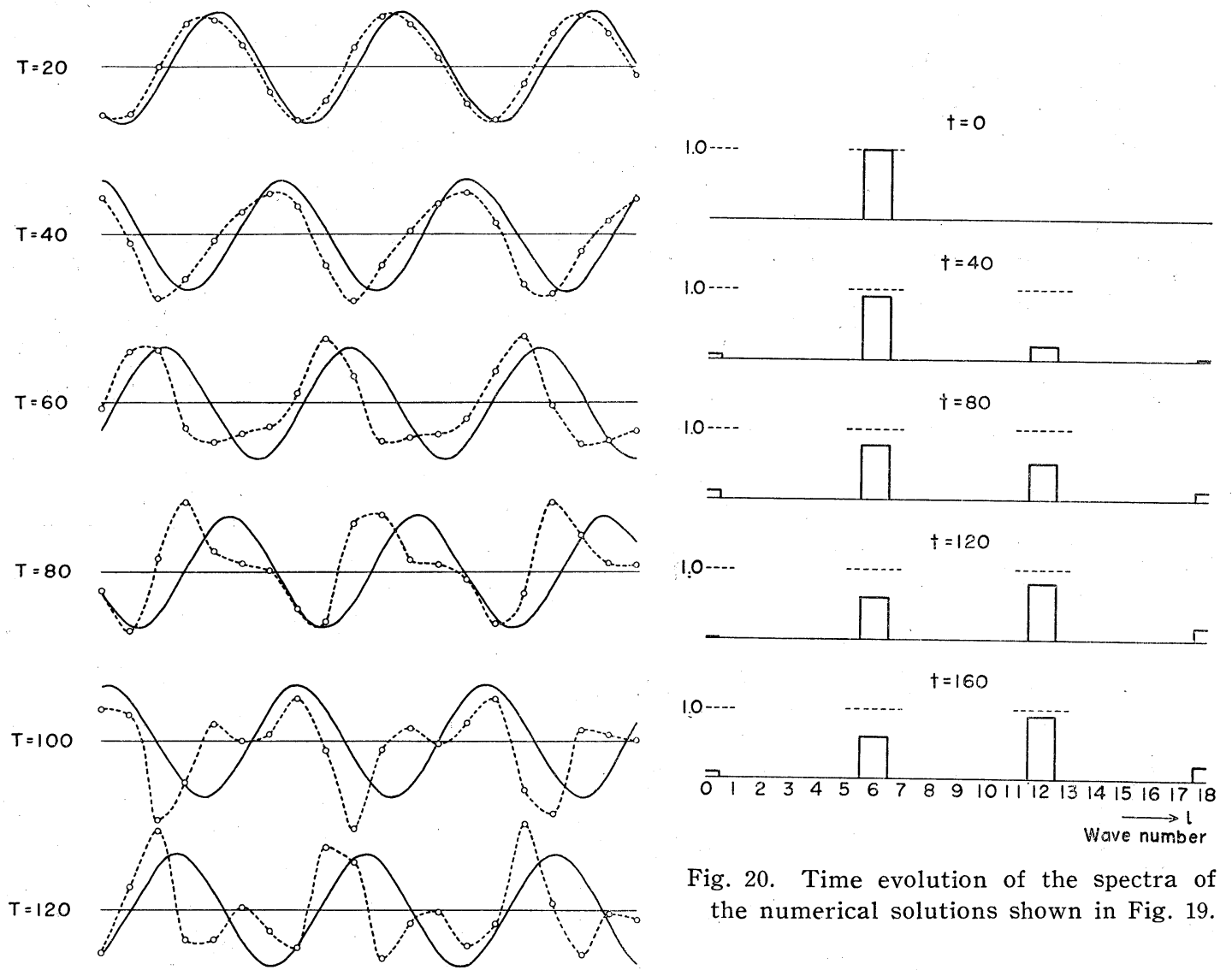

Fig. 20. Time evolution of the spectra of the numerical solutions shown in Fig. 19.

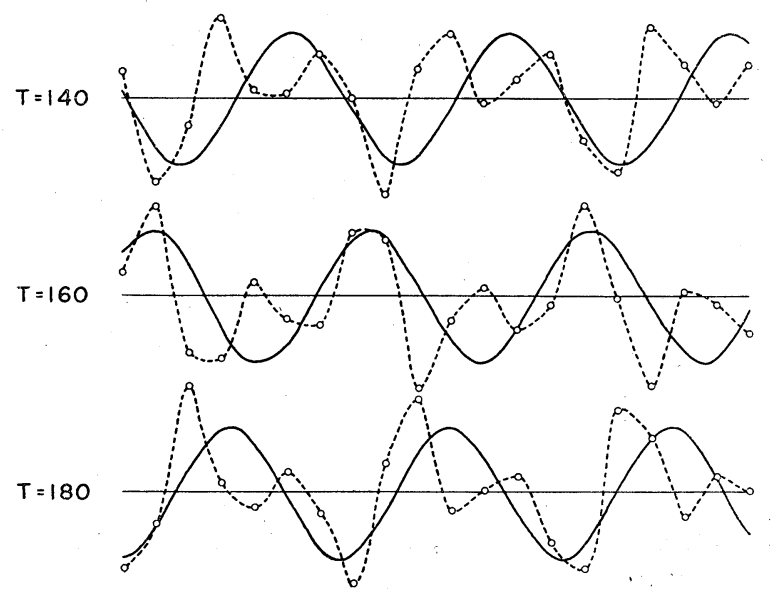

Fig. 19. Time evolution of the pattern. The solid lines are the exact solutions and the dashed the numerical solutions by the $3-3 \mathrm{pt}$ method together with the smoothing operation. 
$T=0$
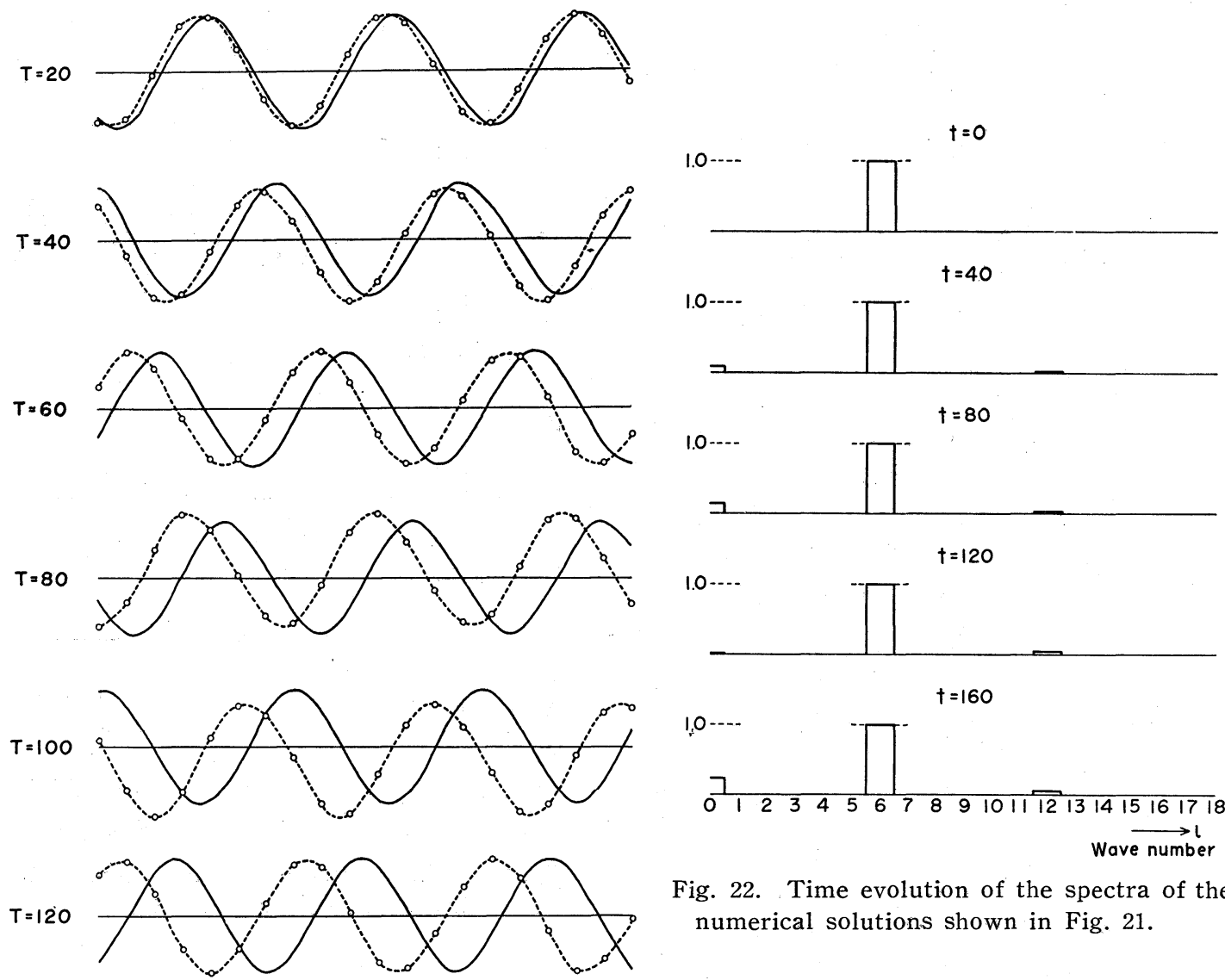

Fig. 22. Time evolution of the spectra of the numerical solutions shown in Fig. 21.

$T=140$
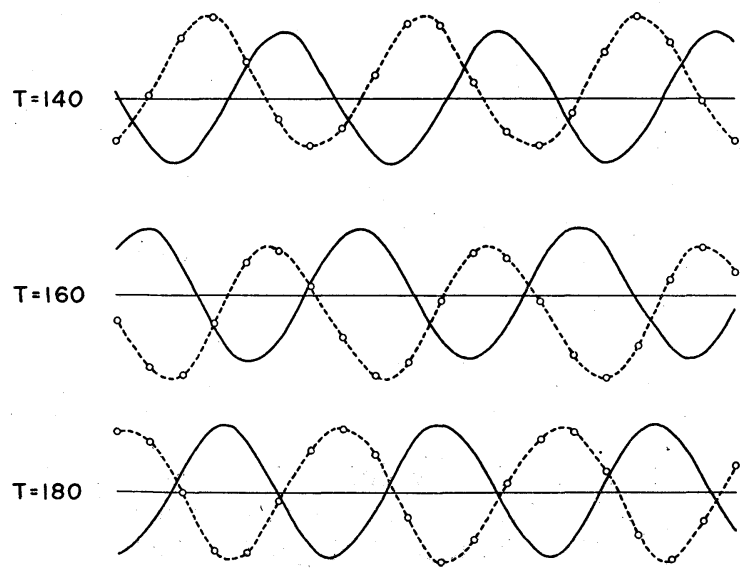

Fig. 21. Time evolution of the pattern. The solid lines are the exact solutions and the dashed the numerical solutions by the $3-3 \mathrm{pt}$ method together with the filtering operation. 

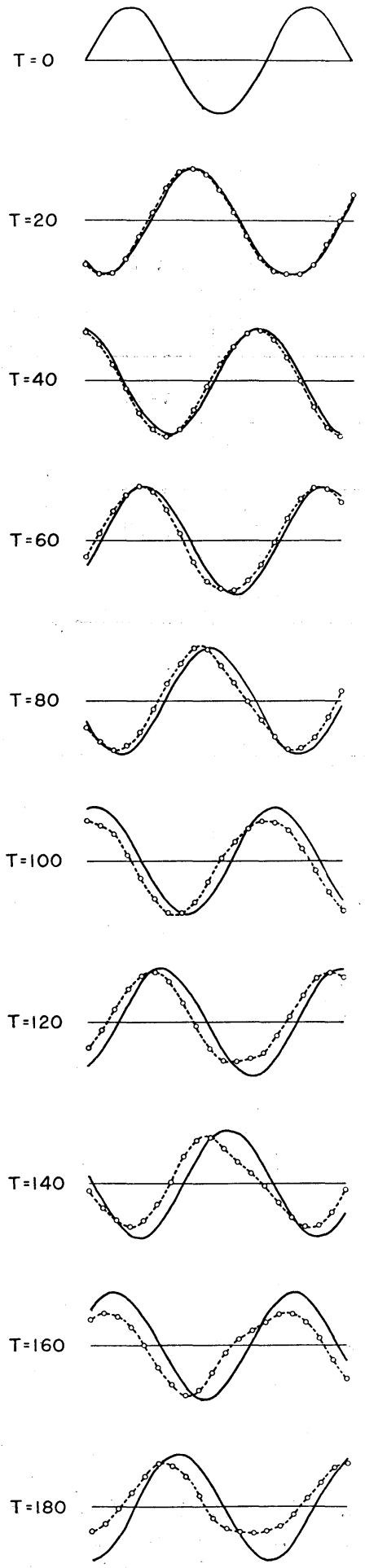

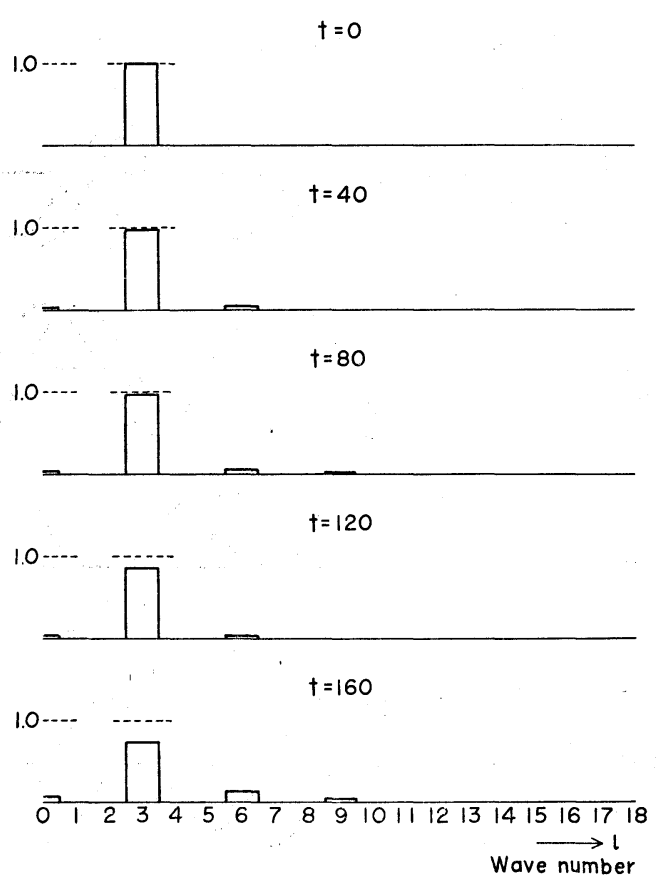

Fig. 24. Time evolution of the spectra of the numerical solutions shown in Fig. 23.

Fig. 23. Time evolution of the pattern. The solid lines are the exact solutions and the dashed the numerical solutions by the 3-3 pt method and by taking half of the gridsize used in the previous test shown in Fig. 16. 
First, the wave solution at every time step is analyzed by the Fourier method and the harmonics higher than a certain wave number, i.e., 8 are cut off, and the remaining harmonics are recomposed into a wave solutions.

The results are shown in Figs. 21 and 22. It is a characteristic feature that the wave form is not changed from the original but the phase of the numerical wave delays clearly from that of the exact wave.

Generally speaking, however, as was pointed out before, the wave components decomposed by the Fourier series have not physical significance themselves and it is not favorable for the practical computation because of the labor it needs. Thus, the filtering process locally applicable is desired.

\section{(e) Refinement of net}

According to the foregoing, the error of the wave number $2 m$ increases with time proportionally to $(m \Delta s)^{3}$ or $(2 \pi / p)^{3}$, where $p$ is the number of gridpoints included in one wavelength. If, therefore, the gridsize $\Delta s$ is shortened, the error would probably be diminished.

In this meaning, we next perform the experiment by making half the gridsize, that is, $\Delta s$ is $150 \mathrm{~km}$, whereas $300 \mathrm{~km}$ previously. Figs. 23 and 24 show the results of time evolution of the numerical and the exact solutions and the variation of spectra.

The production and the development of error have decreased remarkably. But, the refinement of the gridsize is very laborious, because it doubles or triples the number of gridpoints of a net used for computation. Therefore, this means should be taken finally after seeking for all the methods available.

\section{(f) The errors for various scales of waves}

It would be instructive to know the degree at which the error is formed for the various scales of waves.

As shown in Figs. 18 and 25, the error of the wave number $m$ and $2 m$ increases with time step. In the similar manner, we made the calculations with respect to other cases. In Figs. 25 and 26, the deviations of amplitude for the wave number $m$ and $2 m$ are given

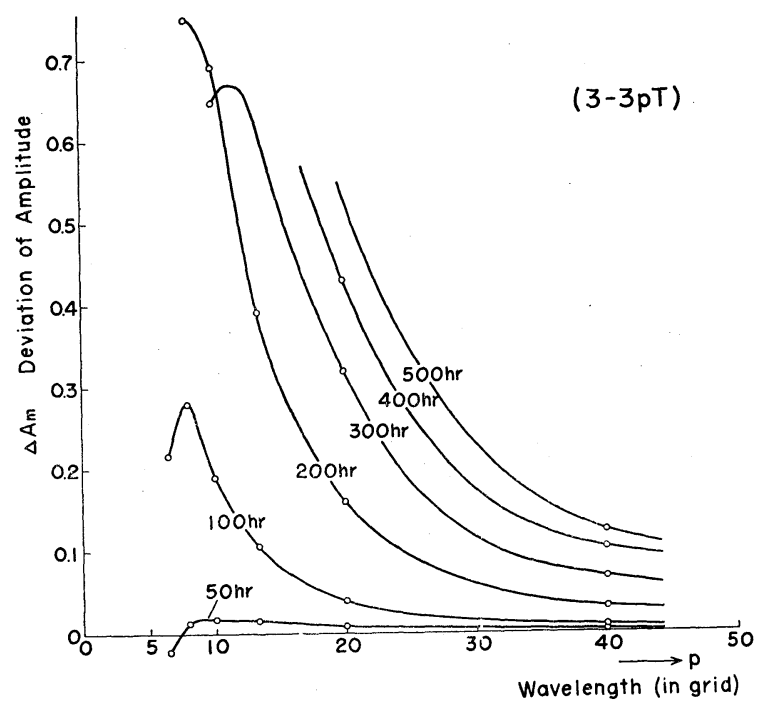

Fig. 25. Deviations of amplitube of the wave number $l$ or $m$ against the wavelength $p$ in unit of gridpoint in the $3-3$ pt scheme. The curves are drawn with the parameter of the forecast time of $50,100,200,300,400$ and 500 hours.

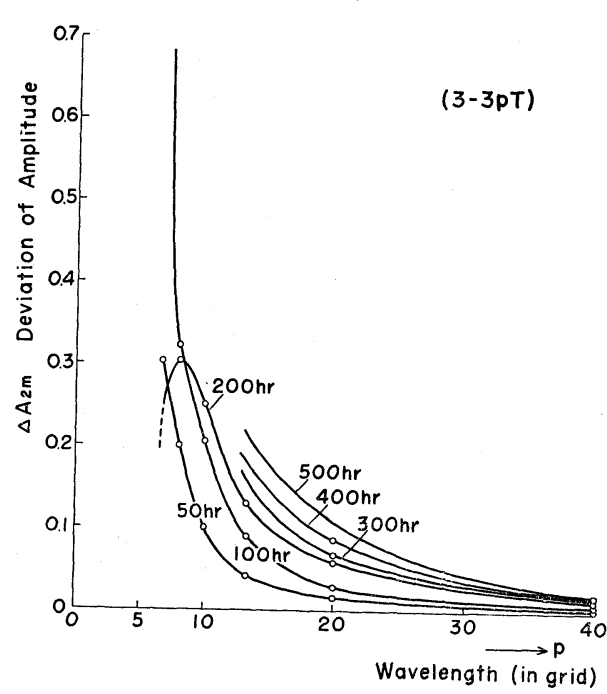

Fig. 26. Deviations of amplitude of the wave number $2 \mathrm{~m}$.

against the number of gridpoints involved in one wave, for the forecast time 50,100, 200, 300,400 and 500 hours. From these figures, we see that the increase of error is roughly proportional to $(2 \pi / p)^{3}$, and that, for instance, in order to forecast $\varphi$ at $500 \mathrm{hr}$ ahead, we must take the grid so that the number of gridpoints involved in one wave is 20 or 30 , 
in the case that the values of $U_{1}, U_{0}$ and the functional form of $U$ are just as assumed here.

(g) The measure of expecting locally the occurrence of error in the 3-point space difference scheme

As was referred previously, the concept of wave is not convenient for the practical purpose, and the Fourier method only gives the artificial wave. Thus, what is wanted is the measure of the scale of waves, which can be locally defined, and which is capable of giving the information where and to what extent the error is formed.

Concerning this matter, we see that the

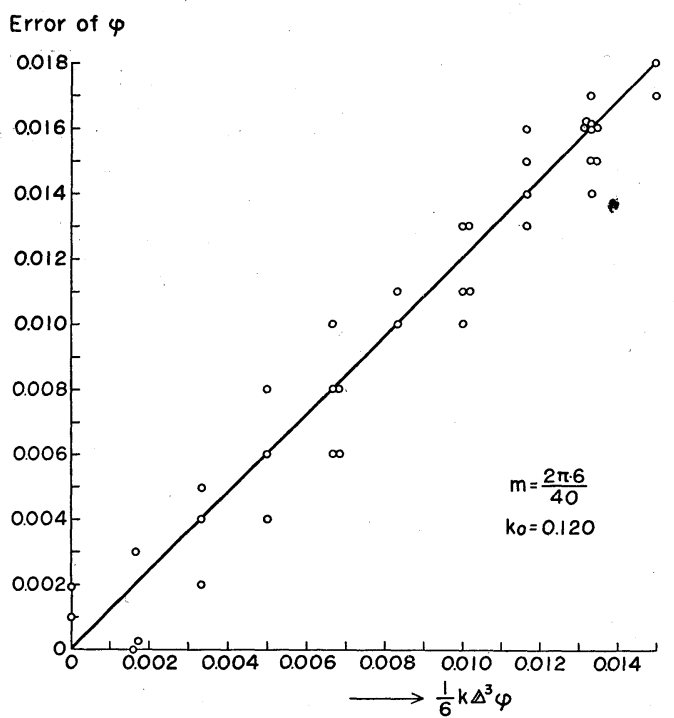

(a)

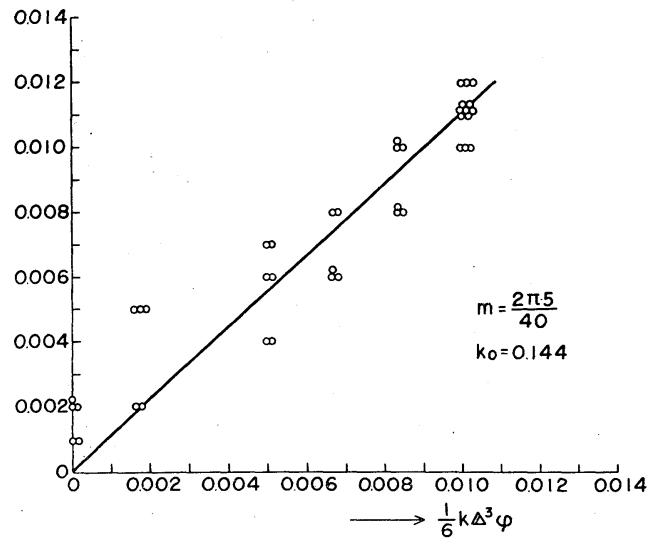

(b) formula (4.15), especially $\varepsilon_{1}$ gives the information about the error. It is not, however, the error of the solution itself but of the equation. But if the residual of equation is large, the error of the solution may also be large. In this meaning, we set up the following quanțity

$$
\varepsilon^{*}=\frac{1}{6} U \Delta t \mid \Delta s \cdot \Delta^{3} \varphi,
$$

where $\Delta^{3} \varphi$ is the third derivative in the difference form defined by

$$
\Delta^{3} \varphi=\frac{1}{2}\left(\varphi_{i+2}-\varphi_{i-2}\right)-\left(\varphi_{i+1}-\varphi_{i-1}\right) .
$$

We compare $\varepsilon^{*}$ with the error of $\varphi$ newly formed during one time step. The results

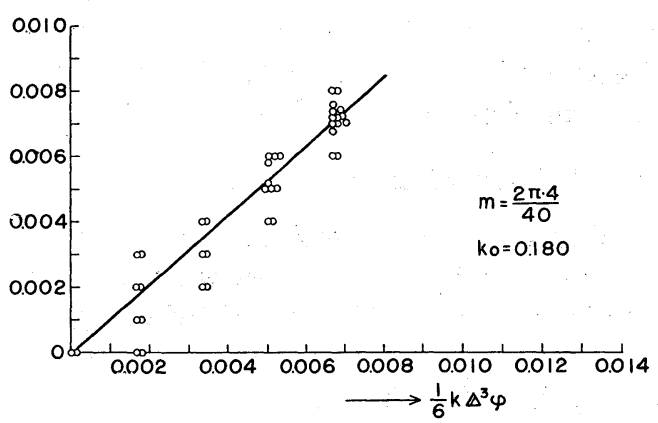

(c)

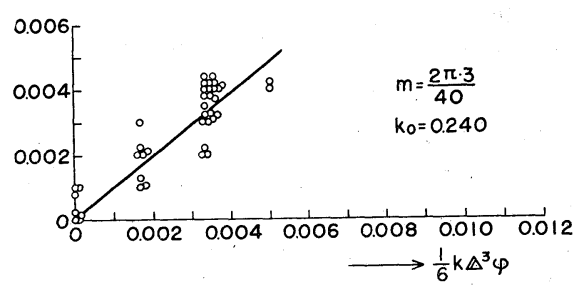

(d)

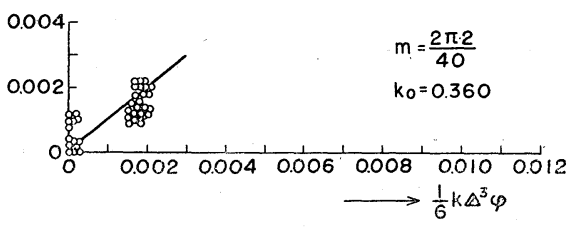

(e)

Fig. 27. The measure to estimate the error $1 / 6 k \Delta^{3} \varphi$ and the error of $\varphi$ actually produced in the case of the $3-3 \mathrm{pt}$ method. 
are shown in Figs. 27's for the various waves, where the ordinate is the error of $\varphi$ and the abscissa the measure $\varepsilon^{*}$. The comparison was made for the same gridpoint for $\varepsilon^{*}$ and the error. It should be noted that $\varepsilon^{*}$ becomes larger with increasing time step. This fact would indicate that the occurrence of error becomes possibly large with time step.

As is seen in Figs. 27, $\varepsilon^{*}$ in the small scale of waves distributes in the wider range from the large values to the small, and the plotted points are fairly systematically lined up, and further, the quantitative relation between $\varepsilon^{*}$ and the error of $\varphi$ is the same for any wavelength and any case.

Thus, it is proved that $\varepsilon^{*}$ may possibly be the measure of the scale of waves and of the error which will be produced. As the practical application, for example, in order to treat the pattern consisted of the various waves, and in order to suppress the waves smaller than the wave of the wavelength of 8 gridpoints, the criterion for $\varepsilon^{*}$ should be $0.07 \sim$ 0.09 .

\section{(h) Conclusions}

The most characteristic thing in this section is that the equation capable of producing many waves is treated. Namely, the interaction of two quantities gives arise the new waves, one is larger and the other smaller. It is then very likely that the small wave involves much error. This process is taking place not only in the non-linear equation but also even in the linear equation, if two quantities multiplied to each other are space dependent. We discussed the error produced in this system of equation and got the following conclusions.

(1) The error occurring in the form of small scale of wave becomes a ruggedness of the resulted pattern, and it develops with increasing time step.

(2) This error does not belong to that due to the computational instability commonly known.

(3) This error comes from the erroneous interaction of two quantities implied in the equation, and the reason lies in the inaccurate approximation of space derivative by the finite difference.

(4) The error increases with decreasing wavelength of the pattern dealt with. In other words, it is related to the number of gridpoints involved in one wave.

(5) We seeked for the measure of expecting locally the occurrence of error. Then, we propose the quantity for 3-3 pt method as in eq. (4.22), for the advection equation. It is shown that this quantity is in a good relation quantitatively with the error of $\varphi$ produced in one time step.

(6) In order to suppress the production of the erroneous small scale of error, the methods of "smoothing" and "filtering" are tested. But both do not give a satisfactory result, though the blowing-up of the error is, to some extent, suppressed.

(7) Just as a trial, the mesh is refined. Then, the result gives us a satisfaction. But it should be taken as the final means after seeking for all the methods available.

\section{Five point space difference method}

\section{(a) The method and examples}

As described so far, the error arising from the approximation of space derivative is much larger than that from time derivative. This is because we usually pay a due attention to the criterion of computational stability and take the small time interval $\Delta t$, but not for the space derivative.

Therefore, it may be natural to presume that if the scheme of approximation of the space derivative is revised, the results would be more improved. Then, instead of the $3 \mathrm{pt}$ method hitherto adopted for approximation to $\partial \varphi / \partial x$, the $5 \mathrm{pt}$ method is taken as

$$
\begin{aligned}
\frac{\partial \varphi}{\partial x} & \Rightarrow \frac{4}{3}\left[\varphi_{i+1}-\varphi_{i-1}-\frac{1}{8}\left(\varphi_{i+2}-\varphi_{i-2}\right)\right] / 2 \Delta s \\
& =\Delta_{5} \varphi .
\end{aligned}
$$

It is readily seen that error produced by this scheme is

$$
\frac{\partial \varphi}{\partial x}=\Delta_{5} \varphi+\frac{1}{30} \Delta s^{4} \frac{\partial^{5} \varphi}{\partial x^{5}} .
$$

The results are shown in Fig. 28. It is really remarkable that the error is very small and there is no possibility to give any computational instability. Further the errors for various scales of waves are shown against the number of gridpoints involved in one wave in Figs. 29 and 30. Comparing these curves with those in Figs. 26 and 27, it is seen that 

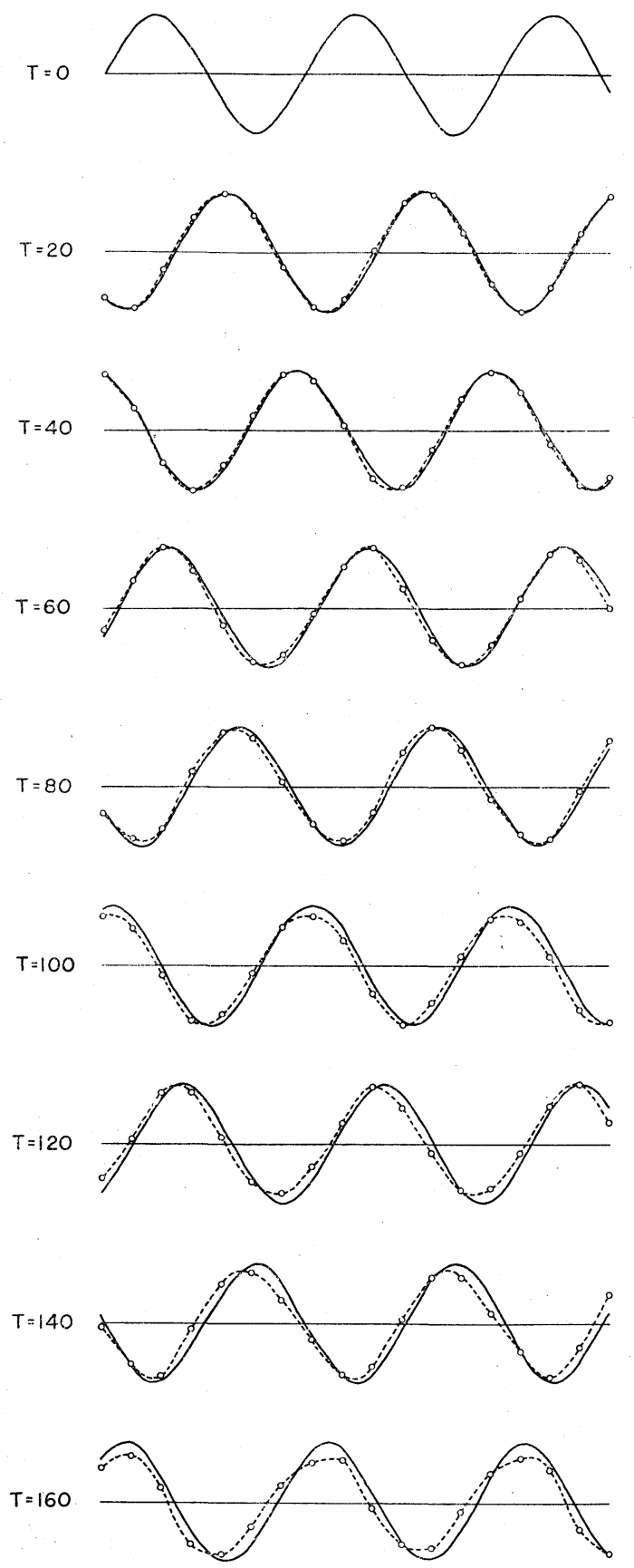

Fig. 28. Time evolution of the pattern. The solid lines are the exact solutions and the dashed the numerical solutions by the $3-5 \mathrm{pt}$ method.

the increase of error is very small by about one-tenth, and that the errors produced at the small scale of waves are especially small than those in the 3 pt method. This implies that small irregularities of the pattern would ap-

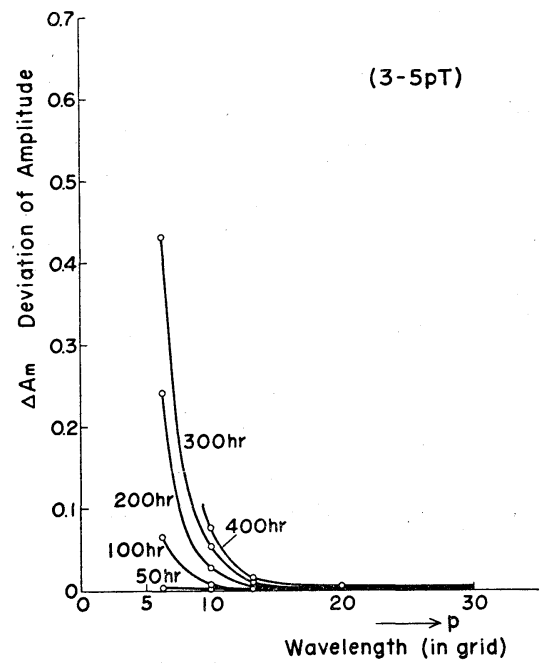

Fig. 29. Deviations of amplitude of the wave number $l$ or $m$ against the wavelength $p$ in the 3-5 pt scheme.

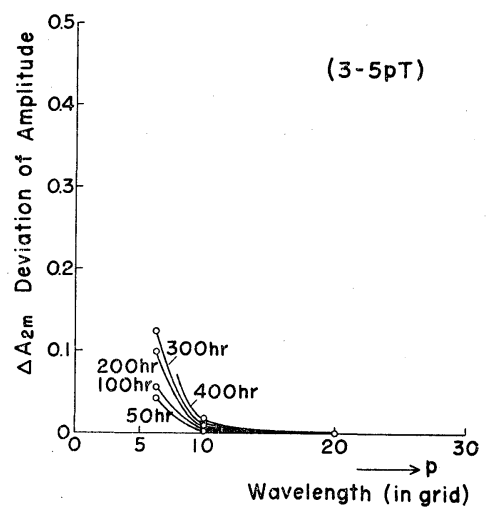

Fig. 30. Deviations of amplitude of the wave number $2 \mathrm{~m}$.

pear in far less extent than in the $3 \mathrm{pt}$ method.

(b) The measure for error

In the similar manner as mentioned in the previous section, the measure of expecting the development of error in this case is given by

$$
\varepsilon^{*}=\frac{1}{30} k \cdot \Delta^{5} \varphi
$$

where

$$
\begin{aligned}
k= & U \Delta t / \Delta s, \\
\Delta^{5} \varphi= & 0.5\left[\varphi_{i+3}-\varphi_{i-3}+\varphi_{i+1}-\varphi_{i-1}\right] \\
& -2\left(\varphi_{i+2}-\varphi_{i-2}-\varphi_{i+1}+\varphi_{i-1}\right) .
\end{aligned}
$$

The practical comparison between $\varepsilon^{*}$ and the error of $\varphi$ formed in one time step is made 


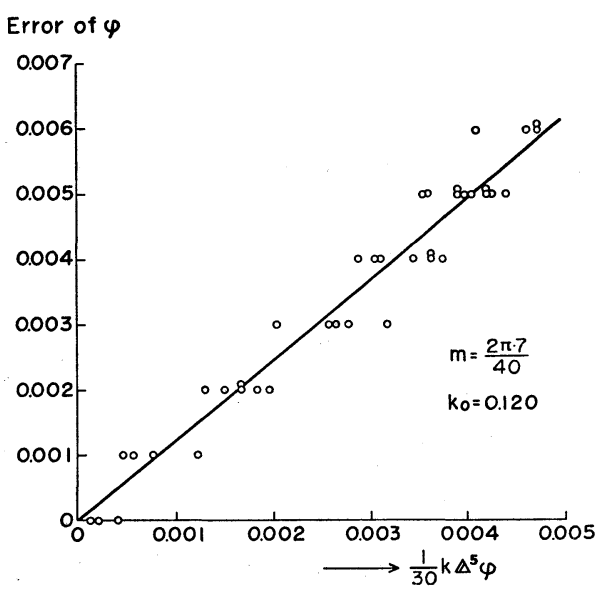

(a)

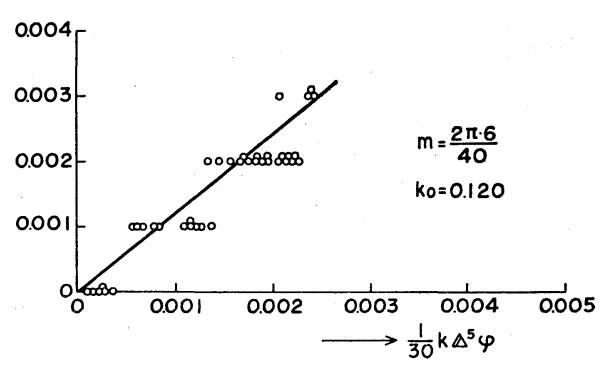

(b)

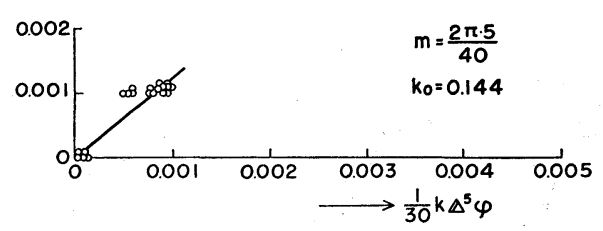

(c)

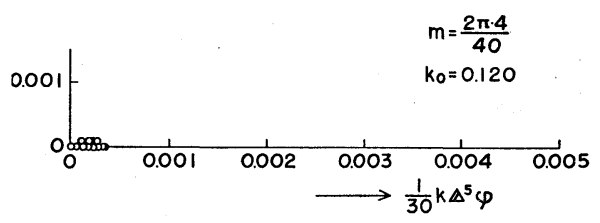

(d)

F1g. 31. The measure to estimate the error $1 / 30 k^{5} \varphi$ and the error of actually produced in the case of the 3-5 pt method.

and the results are shown in Fig. 31, where the scale of ordinate is taken as twice that of Fig. 27. The relation is just the same quantitatively as in the case of the $3 \mathrm{pt}$ scheme.

It is, however, presumed that if we take the quantity, considering (4.13), as

$$
\begin{aligned}
\varepsilon^{*}= & \frac{1}{30} k \Delta^{5} \varphi-\frac{1}{6} k^{3} \Delta^{3} \varphi-\frac{1}{6}\left(k^{2} \Delta^{2} k+k \cdot(\Delta k)^{2}\right) \Delta \varphi \\
& -\frac{1}{2} k^{2} \boldsymbol{\Delta} k \Delta^{2} \varphi,
\end{aligned}
$$

instead of (5.3), the more exact relation may possibly be obtained.

\section{(c) Conclusions}

(1) The 5 pt space difference method gives a remarkably good result.

(2) The measure for error is given by (5.3).

\section{Cutting}

In the practical problem, the pattern treated involves various scales of waves or various kinds of undulation. Therefore, in order to ascertain a certain degree of accuracy, we should remove the small waves from the pattern initially given together with in the course of marching computation.

For this purpose, the quantities given by (4.22) and (5.1) for the $3 \mathrm{pt}$ and the $5 \mathrm{pt}$ methods, respectively are proposed as the measure of the elimination of the small undulation. Practical procedure is described by taking an example of the $5 \mathrm{pt}$ method.

First, the quantity $\varepsilon^{*}$ is calculated at every gridpoint from the pattern dealt with. Second, taking the consecutive 5 gridpoints, we investigate whether or not the absolute values of $\varepsilon^{*}$ at these points exceed a criterion prescribed in advance. The magnitude of criterion determines the degree of accuracy of the predicted pattern. Third, if some points show the excess, the finite difference Laplacian $\Delta^{2} \varphi$ 's are calculated at 5 points, and the largest absolute value of $\boldsymbol{\Delta}^{2} \varphi$ is diminished by reducing the absolute value of $\varphi$ at the point. Thus, the flatening of the pattern $\varphi$ is taking place. The processes from the second mentioned above are applied to every gridpoint. After scanning all the points, we go back to the beginning and continue the same process. In this way, the process is repeated until there exists no gridpoint where $\varepsilon^{*}$ exceeds the criterion.

At the Japan Meteorological Agency, the "cutting" process different from the above is being adopted for the modification of the pattern. (for instance, Saito, 1960). The criterion was proposed by Gambo (1957), that is, 
the absolute, value of $\Delta^{2} \varphi$ must not exceed that of coriolis parameter so that the geostrophic approximation is valid for the differential equation we are treating. The author considers, however, the cutting should be required rather from the numerical point of view as mentioned above.

\section{Sample calculations of $\mathbf{5 0 0}$ hour fore- cast}

As the final summary, we attempted to perform the 500 hour forecast of an arbitrary pattern consisting of multiple waves in the system of one-dimensional linear equation without using the "cutting".

(a) The case not including the friction term

The equation is

$$
\frac{\partial \varphi}{\partial t}=-U(x) \frac{\partial \varphi}{\partial x}+f(x, t)
$$

and

$$
\begin{aligned}
U= & 10+3 \sin 5 n x \quad(\text { in } \mathrm{m} / \mathrm{sec}), \\
\varphi= & \sin 4 n\left(x-c_{1} t\right)+0.7 \sin 6 n\left(x-c_{2} t\right) \\
& +0.3 \sin 10 n\left(x-c_{3} t\right),
\end{aligned}
$$

where

$$
c_{1}=10, \quad c_{2}=12, \quad c_{3}=14 \quad(\text { in } \mathrm{m} / \mathrm{sec})
$$

and

$$
n=2 \pi /(40 \cdot \Delta s), \quad \Delta s=300 \mathrm{~km} .
$$

The time interval $\Delta t$ was adopted as 1 hour. The determinations of the starting values in the marching calculation were all based on the half time interval method. The tests were made with respect to the $3-3 \mathrm{pt}$, the $3-5 \mathrm{pt}$ and the $4 \mathrm{C}-5 \mathrm{pt}$ methods. The results were shown in Fig. 32, 33, 34, at the time interval 40 hours, where the numerical solutions were compared with the analytical and the standard deviations between them were indicated. As seen in these figures, the usual method, i.e., the 3-3 pt method produces much error compared with the $3-5 \mathrm{pt}$ and the $4 \mathrm{C}-5 \mathrm{pt}$ methods. It would be very remarkable that even the slight and delicate undulations of pattern are realized in the numerical results by the $3-5 \mathrm{pt}$ and the $4 \mathrm{C}-5 \mathrm{pt}$
$(3-3 p T)$
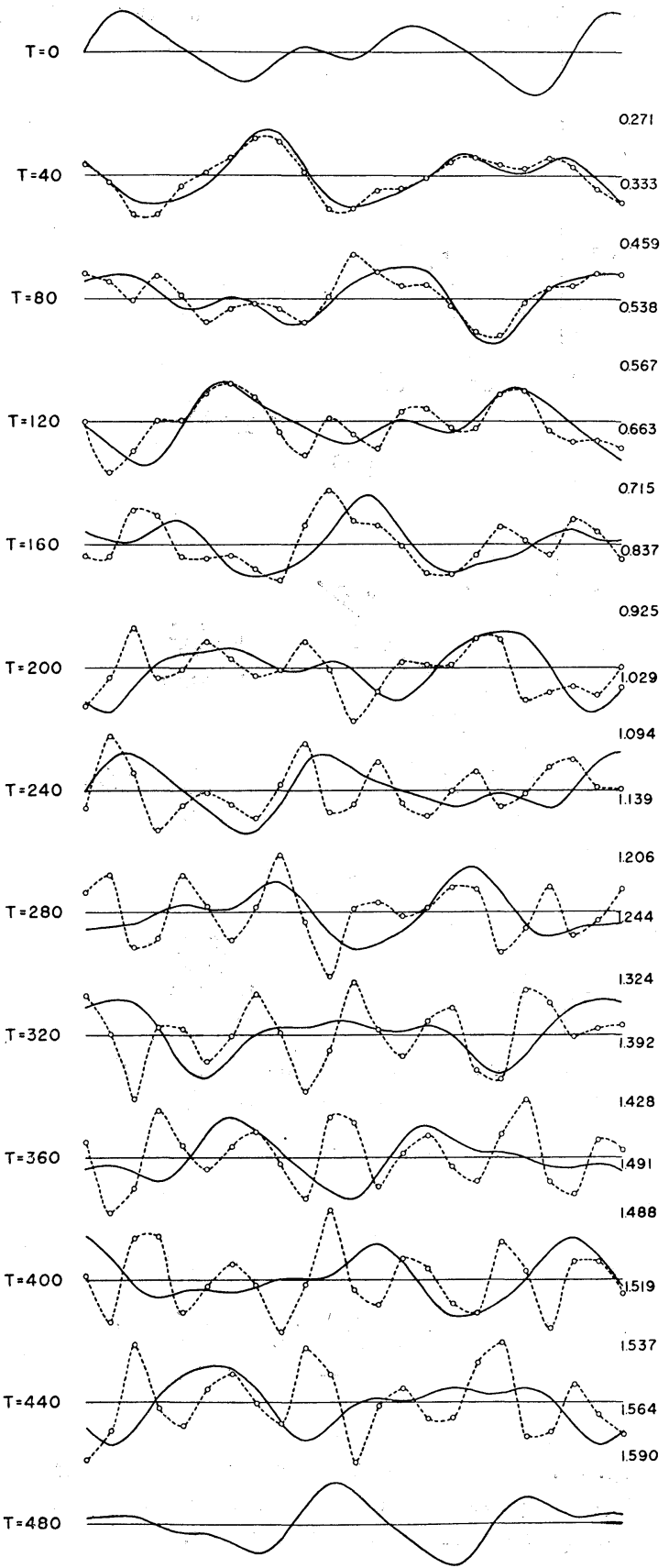

Fig. 32. Time evolution of the pattern at the interval of 40 hours. The solid lines are the exact solutions and the deshed the numerical solutions by the 3-3 pt method. The figures at the right indicate the standard deviations of the numerical from the true solutions at the interval of 20 hours. 
$(3-5 p T)$
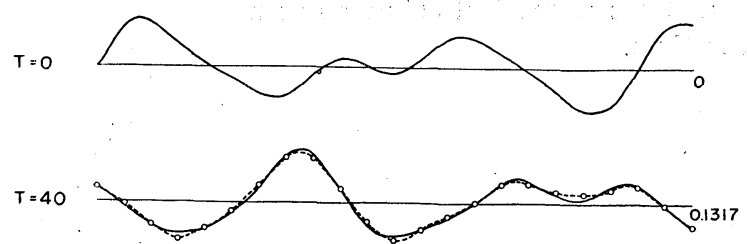

$T=80$

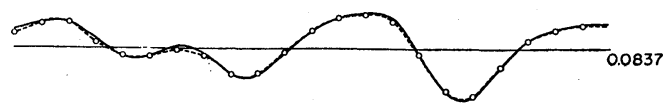

$T=120$
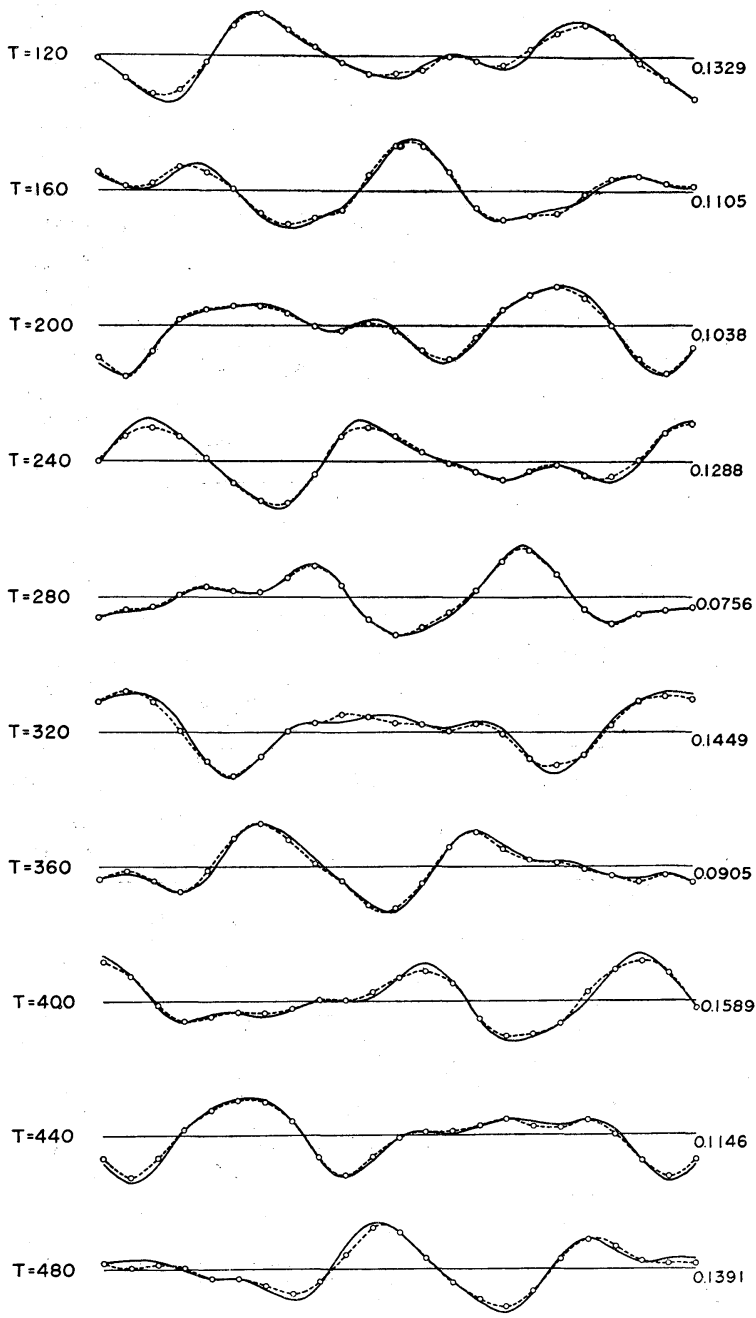

Fig. 33. Same as Fig. 32 but by the 3-5 pt method.
$(4 c-5 p T)$
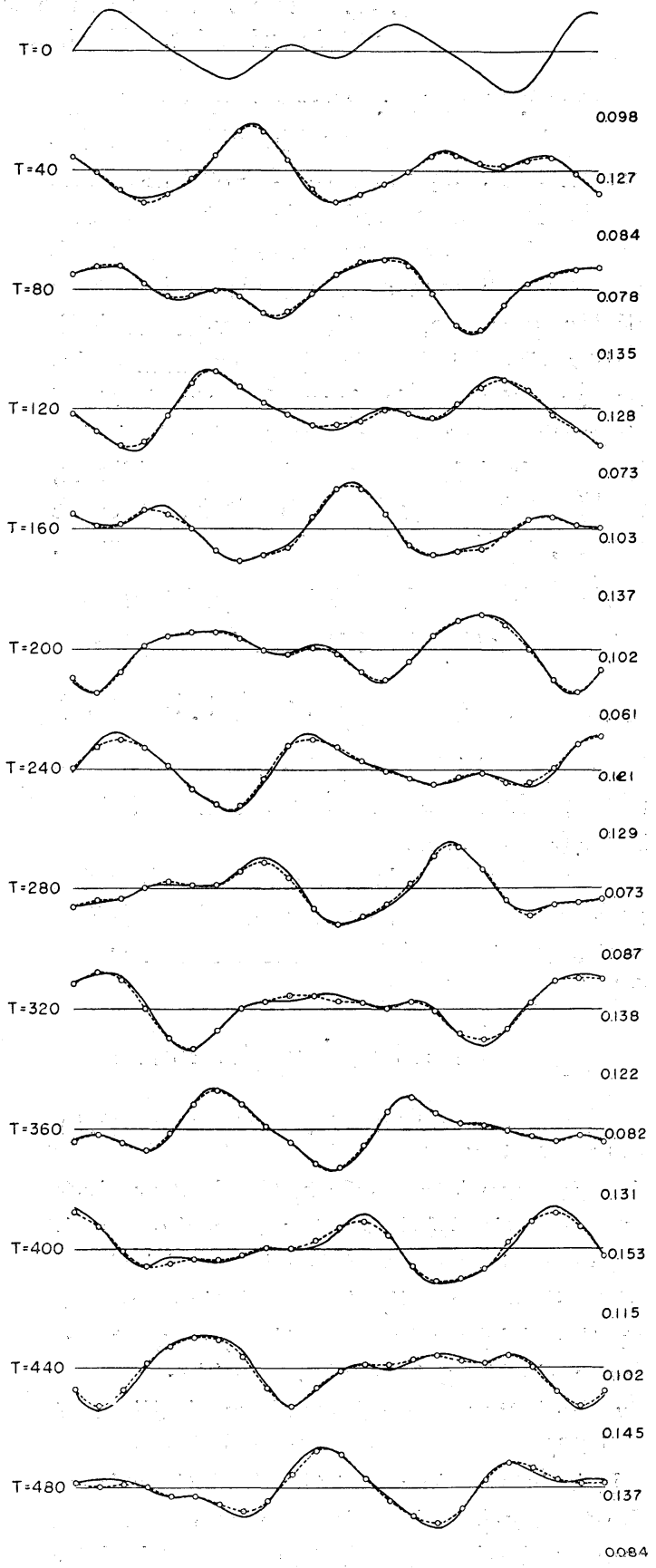

Fig. 34. Same as Fig. 32 but by the $4 \mathrm{C}-5 \mathrm{pt}$ method. 


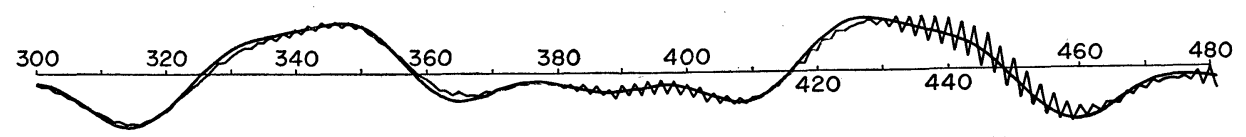

Fig. 35. Time variation of the true and the numerical solutions at a certain gridpoint. Abscissa gives time.

methods. Further, there can hardly be recognized a considerable difference between the $3-5 \mathrm{pt}$ and the $4 \mathrm{C}-5 \mathrm{pt}$ methods.

The interesting thing is that the standard deviations do not grow up monotonically in the case of the composed pattern of many waves, but they show the periodical variation though they gradually increase. From this, it would be understood that the exact evaluation of the deviation in the individual case is very difficult, though the upper bound of it is, to some extent, possible to be estimated in advance.

(b) The case including the friction term

The equation in this case is

$$
\frac{\partial \varphi}{\partial t}=-U(x) \frac{\partial \varphi}{\partial x}+\nu \frac{\partial^{2} \varphi}{\partial x^{2}}+f .
$$

The quantities are the same as those adopted in the previous tests except that $2 \Delta t \cdot \nu /(\Delta s)^{2}=0.008$.

The tests in this case are made with respect to the $3-5 \mathrm{pt}$ and the $4 \mathrm{C}-5 \mathrm{pt}$ methods. The difference formula for the friction term is as follows

$$
\begin{aligned}
\frac{\partial^{2} \varphi}{\partial x^{2}} \Rightarrow & {\left[\frac{4}{3}\left(\varphi_{i+1}+\varphi_{i-1}\right)-\frac{1}{12}\left(\varphi_{i+2}+\varphi_{i-2}\right)\right.} \\
& \left.-\frac{5}{2} \varphi_{i}\right] /\left(2 \Delta s^{2}\right) .
\end{aligned}
$$

As was mentioned before, the former gives the oscillation of the pattern. In the practical test, since the value of the frictional coefficient is small, the oscillation appears at the fairly advanced time, say, at 280 hours (in Figs. 35 and 36). The results by the latter method are shown in Figs. 37 and 38 .

It is interesting that the deviations from the true values in this case become small compared with those in the case not including the frictional term.

\section{Conclusions and future work}

We studied the inherited error in the numerical time integration of one-dimen-
Friction $(3-5 p T)$
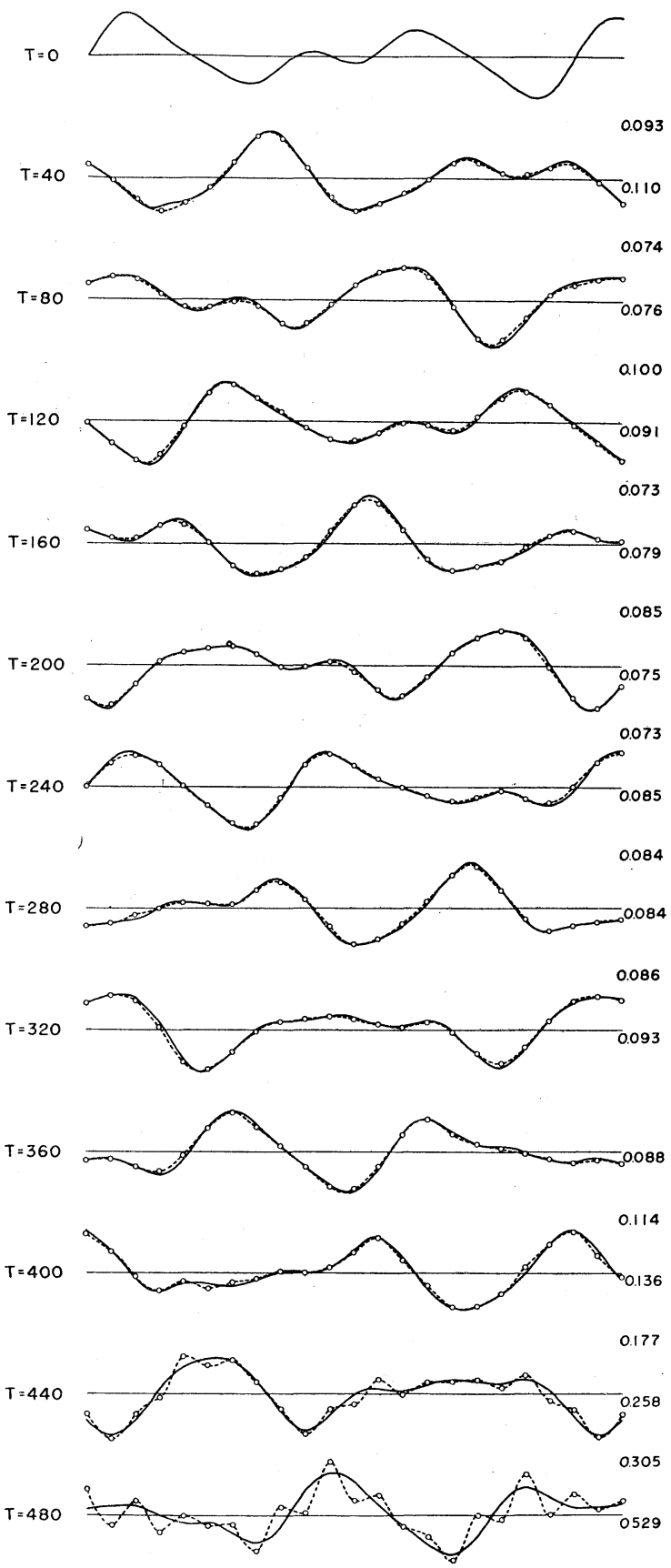

Fig. 36 . 


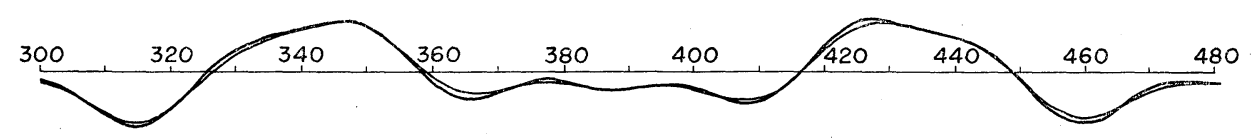

Fig. 37.

$(4 c-5 p T)$
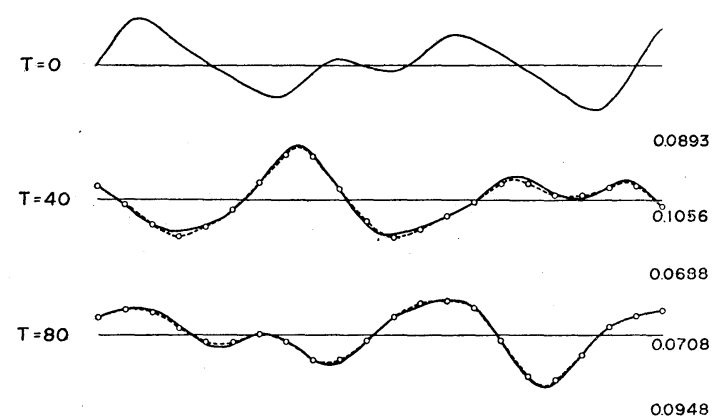

$T=120$

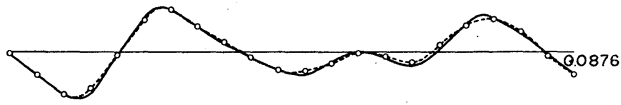

$T=160$
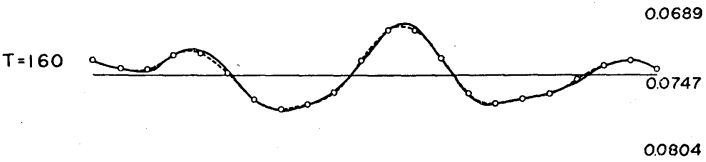

$T=200$

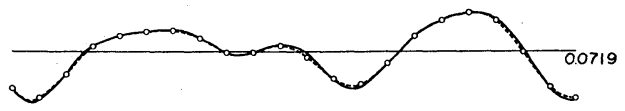

$T=240$
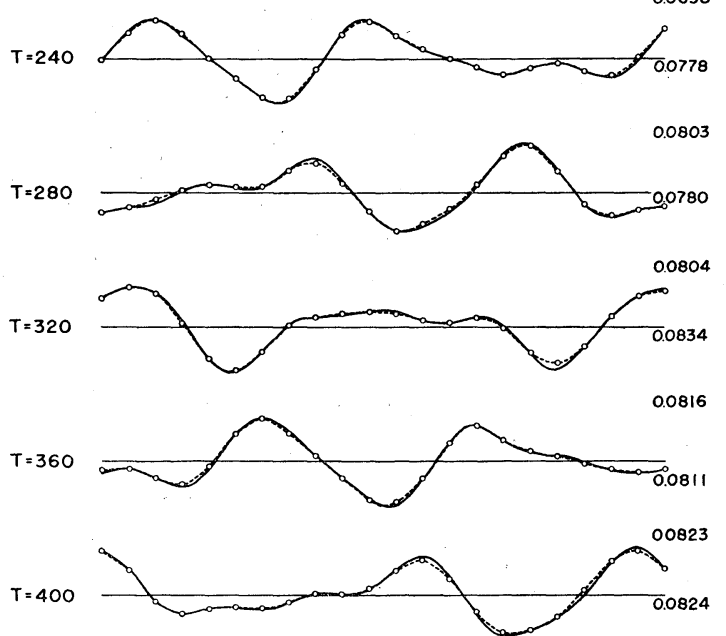

0.0824
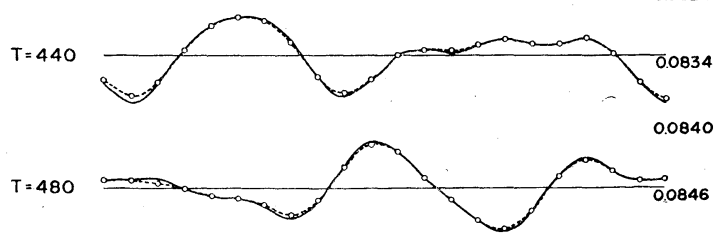

Fig. 38. sional linear equation and the numerical techniques which give the accurate and stable solution. The equation treated involves the advection and the friction terms, where the former includes the case that the advecting speed is variable with space.

The method of comparison of the numerical with the analytical solution is made by the slightly tricky manner, but the conclusion about the information of inherited error is never violated. Namely, the equation which is to be dealt with is added by a term of the function of space and time. First, we assume the functional form of the solution of the equation and, then, the form is inserted into the equation. Thereby, the functional form of the residual term is determined so as to suffice the equation. This method does not provide any aid for obtaining the exact solution of an arbitrary equation. However, in the examination of the numerical method, in other words, in the calibration of the technique, it is enough to give the information about the error possibly produced by the method.

The results of the test show that the space truncation error by the conventional method is especially large compared with the error arising from the time extrapolation. It is usual that we pay a due attention to the time interval because of the existence of the computational stability criterion. However, the space derivative has usually been much roughly treated. The result by the usual difference method indicates that the small scale waves are fictitiously produced by interaction of two quantities which are the functions of space. Further, the growth rate of the small scale errors is great in the usual method, and these errors make the ruggeduess of the resulted pattern.

As a remedy of the conventional 3 gridpoint method with respect to the approximation to space dervative, the 5 gridpoint method is presented and it is tested with the pattern cyclicly undulating on a rigwise space coordinate. It is much remarkable that accuracy of computation is greatly raised up, and there 
exists no apprehension of the computational instability. It seems that the magnitude of the error produced by the 5 gridpoint method is in the same degree with that arising from the approximation by the usual time extrapolation method, i.e., the 3 time step method.

As to the time extrapolation method, the centered difference method, i.e., the 3 time step method has been said to be unstable for the friction term (or the restitutive nature). Thus, the 4 time step method is proposed, which provides the explicit procedure for obtaining the value at the future time step, and gives the stable solution and the same degree of accuracy with the 3 time step method. The merit of this method is general ly applicable to any type of equation.

Further, it is shown that there exists no stable time extrapolation methods using the values at the time steps more than 5 among the explicit schemes. Therefore, in order to raise up the accuracy more, the author considers it necessary to adopt the smaller gridsize and smaller time interval.

It is demonstrated that the magnitude of the error is estimated in advance from the residual of the terms of Taylor series expansion of the difference of the finite difference from the differential equation. In other words, the residual becomes to be the measure by which one can predict the occurrence of the error produced locally in the space coordinate. In connection with it, the limitation of the smallness of the pattern is discussed, which can be treated by the numerical technique employed and under the value of the gridsize and the time increment adopted.

Thus, the most outstanding conclusion obtained in the study is that the 5 gridpoint method gives the much superior numerical result. In the present practical computation, it dose not make any trouble because we adopted the ringwise grid. If one wants to apply it to the general problem, however, it turns out to be a serious problem how to treat the values at the gridpoints next to the boundary. Therefore, it should be the future work to devise an appropriate method to overcome it. Further, the discussion about the inherited error in general has not yet been completed so far. There remains the possibility that the non-linearity of the equation might cause the computational difficulty which has not been experienced.

\section{Acknowledgement}

The author wishes to express his thanks to Prof. S. Syōno, Tokyo University, who gave him the helpful suggestions and encouragement, and to Dr, H. Itoo, the Japan Meteorological Agency, who cordially gave him favours to use the IBM 704 . He also wishes to thank Mr. Magata, the Meteorological Research Institute of Tokyo, Mr. Matsuno. Tokyo University; and Mr. A. Arakawa, the Japan Meteorological Agency, for their encouraging and stimulus discussions. Finally, the author wishes to thank late Kawata for reading the manuscript.

\section{References}

1. Arakawa, A., 1960: (to be published)

2. Benton, G. S., 1958: Some comments on the numerical integration of the vorticity equations and related equations. Tellus, 10, 3 , 313-325.

3. Gambo, K., 1957: The scale of atmospheric motions and the effect of topography on numerical weather prediction in the lower atmosphere. Pap. Meteor. Geophys., 8, 1-24.

4. Hinkelmann, K., 1959: Ein numerisches Experiment mit den primitiven Gleichungen.

5. Magata, M., 1957: On the truncation errors in numerical prediction. Meteor. Res. Inst. Papers in Meteor. \& Geophys., 8, 133.

6. Miyakoda, K., 1959: The method of numerical time integration of vorticity equation involving the friction term. J. Meteor. Soc. Japan, 37, 10-22.

7. Miyakoda, K., 1960: Numerical calculations of Laplacian and Jacobian using 9 and 25 gridpoint systems. J. Meteor. Soc. Japan, 38, 2, 94-106.

8. Platzman, G. W., 1954: The computational stability of boundary conditions in numerical integration of the vorticity equation. Archiv für Meteor. Geophys. Bioklim., A 7, 29-40.

9. Reiser, H., 1957: On the introduction of centered finite difference quotients in atmospheric prediction models. Studies in Numerical Weather Forecasting, by staff members of research division, Deutscher Wetterdienst, Frankfurt/M, Germany, Tech. Rep.

10. Richtmyer, R. D., 1957: Difference methods for initial-value problems. Interscience Publishers, Inc., New York.

11. Saito, N., 1960: The test of the forecast of 
lower atmosphere by four-level geostrophic model. Tech. Rep. JMA No. 3, 35-48.

12. Shuman, F. G., 1955: Method of designing finite-difference smoothing operators to meet specifications. JNWPU Tech. Memo. 7, June.
13. Thompson, P. D., 1955: Reduction of truncation errors in the computation of geostrophic advection and other Jacobians. JMWPU Tech. Memo. 1.

\section{一次元線型方程式の時間数值積分の方法とその相続誤差}

都田 菊郎

(東京大学理学 部)

一次元線型の偏微分方程式を数值的に解く方法について, 解の精度, 安定性和よび相続誤差を検討した。第 1 亿時 間外㨀法について議論し，3 点法拈よび 4 点法の一種が満足のゆく結果を与をることを結論した。

第 2 飞空間微分を差分で近似する方法について，3 点法执よび 5 点法について調べた。この場合取扱う方程式は移 流方程式で, 特に流速は空間の函数である。すなわち方程式は 2 ケの空間函数の積を含み，その結果，解としてはそ の相互作用として新しい波を生ずる。このような方程式を，従来一般に用いられている時間，空間についての「3-3 点法」で数值的飞解くときは，新しく発生する波飞誤差が多く，それが解のパターンに虚偽の小さな波を与える。一 方「3-5 点法」では安定で極めて精度の高い解が得られること，特に，虚偽の小さい波の発生が著しく少いことが判 つた。また各方法による䛊差の発生を推定するための目安になる量を提案する。これは微分方程式と差分方程式の差 を Tayler 展開して得るもので, 取扱う量が空間的飞変動が大きい程, 誤差が多くなることを量的と表現したものて ある。最後に以上に得た方法执よび情報をもとにして，任意のパターンの 500 時間予報を試みた結果を示す。 